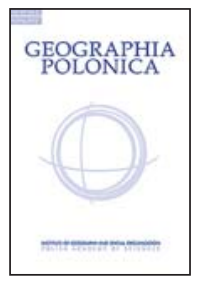

\title{
HOW SENIORS LIVE FROM AN ECONOMIC, HEALTH, SOCIAL AND EMOTIONAL POINT OF VIEW? MULTIDIMENSIONAL REVIEW OF THE QUALITY OF LIFE OF SENIORS IN EUROPE
}

\author{
Marcela Káčerová ${ }^{1}$ (ID - Jana Ondačková ${ }^{2}$ \\ ${ }^{1}$ Faculty of Natural Science, Department of Economic and Social Geography, \\ Demogeography and Territorial Development \\ Comenius University \\ Mlynská dolina, Ilkovičova 6, 84215 Bratislava: Slovakia \\ e-mail: marcela.kacerova@uniba.sk (corresponding author) \\ ${ }^{2}$ Ministry of Agriculture and Rural Development of the Slovak Republic \\ Dobrovičova 12, SK-812 66 Bratislava: Slovakia \\ e-mail: jana.ondackova@land.gov.sk
}

\begin{abstract}
Quality of life is an extraordinarily multidimensional term. It includes both objective and subjective factors. This article reviews the quality of life of an extremely sensitive group - people over the age of 65, based on data from the pan-European SHARE survey (Survey of Health, Ageing and Retirement in Europe). The survey revealed the disparities in the quality of life of seniors regarding material, health, social and emotional dimensions in 16 European countries. According to the European survey of the evaluation of the quality of life of seniors, those living in Western and Northern European countries are more satisfied with the quality of their life. Generally, it has become apparent that quality of life is interlinked with the institutional framework of the country, family support and individual approaches. Countries in Southern and Eastern Europe have lower values in individual dimensions as well as in the aggregate quality of life index.
\end{abstract}

\section{Key words}

quality of life $\cdot$ senior - Europe $\cdot$ material, health, social and emotional dimension

\section{Introduction}

The demographic behaviour of the European population in the second half of the 20th and the beginning of the 21 st century is characterized by significant changes manifested in all countries. They are reflected in a number of population processes and structures which are typically interconnected in their development. These changes have been most 
strikingly manifested in a drop in fertility, changes in family behaviour, and changes of population age structures (Káčerová, Ondačková, \& Mládek, 2014). The growing number of seniors in the population brings along a number of challenges that society must face in terms of economics, health-care and social issues. Recently, special attention has also been paid to the quality of life of this group of the population.

Nevertheless, due to historical reasons (gradual incorporation of the concept of quality of life into the scope of research of individual branches of science) and a high level of complexity, no standard definition of the term "quality of life" has thus far been elaborated. Quality of life has gradually become the focus of social, health, economic, and geographic sciences. It includes both objective and subjective factors and involves numerous indicators that reflect its multidimensional nature. This stems from the fact that human life as such is complex and multifaceted and has multiple dimensions that may overlap or involve a complex network of internal interdependencies.

The World Health Organization (WHO) sought to reflect this diversity in its definition that can be deemed quite comprehensive. It defines quality of life as "individuals' perception of their position in life in the context of the culture and value systems in which they live and in relation to their goals, expectations, standards and concerns. It is a broad ranging concept affected in a complex way by the person's physical health, psychological state, level of independence, social relationships, personal beliefs and their relationship to the salient features of their environment" (WHO, 1997).

Changes in economic activity, loss of social roles or physical strength are important circumstances in people's lives that significantly impact on their quality of life. Thus, it can be said that age is a parameter that significantly contributes to such modifications. Objective life circumstances and subjective perception (satisfaction) are differentiated by individual age categories within the population. Therefore, the aim of this study is to analyse the quality of life of an extremely sensitive and a growing group, i.e. people over 65. For that purpose, this study adopts (with some modifications) the Index of Well-Being in Older Populations developed by the Population Reference Bureau. The index includes 12 indicators that allow for a comprehensive assessment of the material, health, social, and emotional domains of quality of life. The aggregate quality of life index provides a synthetic view of a population's well-being. Our study is a unique attempt to evaluate the quality of life of the people of sixteen European countries based on a single methodology and data from the pan-European SHARE survey. However, in addition to being defined by personal subjective and objective preferences, the well-being of seniors is influenced by the existing social, economic and institutional frameworks of the home countries of individual respondents. As a result, our goal was to also examine the geographic heterogeneity of individual domains of wellbeing and examine how these factors affect different dimensions of the perceived level of quality of life. Special focus was placed on differences in the quality of life between genders due to the fact that the impact of different activities of men and women in individual stages of their lives (education, employment, social roles and specific diseases) and differences in values attached to individual domains by men and women with respect to their overall quality of life appear to be significant.

\section{Quality of life in old age}

To evaluate the well-being of an older population is a challenging undertaking in one more respect: the senior population is an extremely heterogeneous group and encompasses a very specific part of the population (Mühlpachr, 2004). Considering the complexity of the concept and the existence of different disciplinary perspectives, it is not surprising that there is no agreement on how to define and measure quality of life, and no theory 
of the quality of life in old age (Walker, 2005). Several studies point to a specific perception of well-being in older populations. The study by Mastekaasa, Moum, and Næss (1988) suggests that seniors are more satisfied with their lives compared to others, despite the fact that a smaller number of seniors reported that they were happy. According to Campbell, Converse, and Rodgers (1976) life satisfaction usually declines by the age of 50 or 60 , and subsequently increases slightly or remains stable. The study by Netuveli and Blane (2008) also supports this view. The satisfaction curve increases from about age 50 , then peaks at age 68 and declines afterwards. The level of well-being tends to return below the levels corresponding to age 50 as late as at the age of 86 .

The quality of life of the older population has been the subject of a number of different studies in terms of depth (both particular and complex). The most popular research efforts into the quality of life of the senior population include studies focusing on the health of seniors. Naturally, this was due to the availability of data from surveys conducted on a European and national scale. However, it is clear that the significance of the subjective perception of health is growing (Payne, 2005; Uher, 2014) since seniors are more sensitive to this domain of well-being compared to the younger population (Westerhof, Dittmann-Kohli, \& Thissen, 2001). The prevailing scholarly consensus is that a subjective evaluation of health is a more plausible indicator of health than morbidity and mortality (Walker, 2005). This domain is especially significant among male seniors (Aurelia \& Baldazzi, 2002). Farquahar (1995) reported that poor health was the factor most frequently reported by British seniors as the reason for the negative evaluation of their lives. In particular, seniors mention the loss of mobility, higher sickness rates, pain or depression. Similarly, physical health and functional ability were reported as two of the four most important "ingredients" of quality of life by Spanish seniors (Fernández-Ballesteros, 2002), where health was considered the most important factor by as many as $78 \%$ of the respondents. Moreover, several authors have arrived at the conclusion that the development of certain limitations and depression negatively impacts other domains of life and correlates closely with the overall quality of life (Demura \& Sato, 2003; Orfila, Ferrer, Lamarca, Tebe, Domingo-Salvany, \& Alonso, 2006; Dragomirecká et al., 2008; Netuveli \& Blane, 2008).

Apparently, the social dimension is also a very significant contributor to the quality of life of seniors. While most indicators of a negative evaluation of quality of life were related to health limitations (although the maximum negative evaluation was loss of family), the social dimension was evaluated as highly positive. Seniors evaluated their well-being as positive based on factors such as family, activities and social contact, while health was considered as the fourth most important dimension (Farquahar, 1995). Similarly, the greatest concern related to a decline in the quality of life of British seniors pertained to the loss of friends and loved ones (Barnes, Taylor, \& Ward, 2013). This was reaffirmed by another British survey showing that $81 \%$ of senior citizens cited social relationships as the best factor contributing to their well-being, while 50\% reported poor health as the key factor impeding their quality of life (Bowling \& Gabriel, 2004). Based on a survey of the Korean population conducted by Kim and Kim (2003), seniors enjoying the support of their families and friends, and who are active in paying back their support to their families, generally achieved the highest quality of life scores. Similar findings were reported by Haski-Leventhal (2009) who examined the relationship between the well-being of seniors and their involvement in volunteer activities. Volunteers not only enjoyed better health, they were also more satisfied with their lives. The connection between health (specifically depression) and lifestyle was also the subject of research conducted by Demura and Sato (2003). Using the ANOVA statistical test, they concluded that depression is related to the age, the number 
of friends, family, and employment, and for male seniors, volunteering.

Walker (2005) pointed out that the most common empirical associations with quality of life and well-being in old age are good health and functional ability, a sense of personal adequacy or usefulness, social participation, intergenerational family relationships, the availability of friends and social support, and socioeconomic status (including income, wealth and housing). While there are common associations with quality of life and wellbeing, it is clear that subjective self-assessments of psychological well-being and health are more powerful than objective economic or socio-demographic factors in explaining variations in quality of life ratings.

In Europe, comprehensive studies on quality of life have been conducted covering a broad spectrum of aspects of life. However, these studies were not focused on the senior population but covered a wide range of ages. The so-called Sarkozy Commission Report, published in the aftermath of the 2008 general social and economic crisis, was quite inspirational. It was one of the most notable references for a general conceptualization of the measurement of economic performance and social progress (Annoni, Weziak-Bialowolska, \& Dijkstra, 2012). The report focused on the objective and subjective components of quality of life. The subjective components included cognitive evaluation of one's life, hedonistic experiences, happiness, satisfaction, positive emotion (joy, pride, satisfaction) as well as negative emotions (such as pain, fear, delusions). Quality of life is related to both economic resources, such as income, and non-economic aspects of peoples' lives (what they do and what they can do, how they feel, and the natural environment in which they live). The levels of quality of life that can be sustained over time depend on whether stocks of capital that matter for our lives (natural, physical, human, social) are passed on to future generations (Stiglitz, Sen, \& Fitoussi, 2009).

Well-being 2030, co-funded by the European Policy Centre and the European Commission, is another important project related to quality of life. It is based on qualitative research in eight Member States - Estonia, Germany, Greece, France, Poland, Romania, Sweden and the UK, and aims to determine how quality of life can be measured by analysing the potential of social policies, including employment, health, education, migration and measures to address inequality and poverty in Europe. It focused on three groups of the population: (18 to 29 ); (30 to 44$)$; and $(45+)$ and considered their economic situation. The authors of the project arrived at the conclusion that income level, employment, health, and education strongly impact life satisfaction but they did not identify the most important factor. (European Commission, 2011).

Developing a globally applicable model was also the goal of the EU-funded project entitled Ageing Well: European Study of Adult Well-Being (ESAW). The authors of the research, which was carried out in Austria, Italy, Luxembourg, The Netherlands, Sweden and the UK, arrived at five key components of quality of life: physical health and functioning, cognitive efficacy (self-resources), material security, social support resources and life activity, with an emphasis on the subjective perception of these domains. On a global scale, the highest impact on life satisfaction was material security, followed by health.

A number of projects around the world (Eurofound - Quality of Life Survey, National Accounts of Well-Being, the Canadian Index of Wellbeing, Enhancing Wellbeing in an Ageing Society (EWAS) in New Zealand, The University of Michigan Health and Retirement Study (HRS) in the USA, and the Australian Unity Wellbeing Index) strive to achieve a synthetic evaluation of the quality of life of a population with a special focus on different age groups. The diversity of approaches adopted by individual projects stimulates discussion on the development of a set of composite indices, the suitability of instruments/indicators for monitoring quality of life and the areas of their application in scientific research and policy making. Each of these projects has 
its own justification relative to its objective and availability of data.

\section{Methodology}

Most of the previous studies on well-being arrived at the following set of quality of life domains: economic status, environment (material well-being), physical and mental health, independence, social relationships, family relationships, spiritual and personal activities. Since we believe that material well-being is closely related to the economic status of seniors, these indicators should represent one domain. Likewise, independence (limitation of activities) corresponds to health, or more specifically, to physical condition. On the other hand, mental condition affects emotional experience, perceived satisfaction and future outlook. Similarly, family relationships represent a specific case of social contact and are therefore evaluated jointly.

Based on the above domains, we adopted the Index of Well-Being in Older Populations as the starting point of our research. The index was developed by a team of scientists from the Population Reference Bureau and the Stanford Center on Longevity (PRB, 2011). In its final form, the quality of life index comprised four dimensions that included 12 indicators. Our effort to increase the number of indicators (to cover more quality of life aspects of the senior population) was limited by the questions included in the SHARE survey (or sometimes by the weak representation of the respective answers, e.g. a poor response rate for questions about the number of available rooms). The original well-being index was modified and healthy life years were used instead of average life expectancy; the calculation of the aggregate of quality life index was also adjusted (see below).

The data for our article come from SHARE, the Survey of Health, Ageing and Retirement in Europe that has been carried out in Europe since 2004 in 21 countries over four waves. In this survey, data were collected by personal interviews between an interviewer and the respondent and recorded in questionnaires electronically (CAPI) and on paper (so-called Drop-Offs). Respondents were individuals older than 50. The survey has created a longitudinal database of demographic, health, economic, and social information at the level of both individuals and the entire household. In this study, we used data from the 4th wave of SHARE carried out in 2011-2012 with 16 participating countries (Austria, Germany, Sweden, Netherlands, Spain, Italy, France, Denmark, Switzerland, Belgium, Czech Republic, Poland, Hungary, Portugal, Slovenia, Estonia).

The dimension of material well-being was covered by median household income per capita, and percentage not in absolute poverty. Median household income per capita was established based on the question: How much was the overall income, after tax, that your entire household had in an average month in last year? and the question: Does anyone else live in this household? was used to determine the number of members living in the household. To allow for comparability, the median income in individual countries was converted to the international dollar using purchasing power parity. Penn World Table 7.1 (Heston, Summers, \& Aten, 2012) was used for the purpose of the above conversion. The values for the countries using currencies other than the Euro were first converted from the original currency using the coefficient specified in the SHARE database, and the obtained figures were later converted to US dollars in accordance with purchasing power parity. The percentage not in absolute poverty was calculated based on the Low income cut-offs after taxes values for a medium-size community in the relevant year (Statistics Canada, 2014). The after-tax income was converted to the international dollar with purchasing power parity and resulted in the following absolute poverty levels:

- 1-person household - \$13,864,

- 2-person household - \$16,875,

- 3-person household - \$21,012,

- 4-person household - \$26,215,

- 5-person household - $\$ 29,851$,

- 6-person household - \$33,106,

- 7-person household - \$36,360. 
The health well-being dimension was characterised by the following 5 variables: percentage with no disability, percentage with no difficulty taking medications (living independently), percentage with no difficulty walking a short distance (no functional limitations), healthy life-years at age 65 , and percentage not obese. The percentage with no disability was calculated based on the answer to the following questions: Here are a few more everyday activities. Please tell me if you have any difficulty with these because of a physical, mental, emotional or memory problem. Again exclude any difficulties you expect to last less than three months. 1. Dressing, including putting on shoes and socks 3. Bathing or showering 4. Eating, such as cutting up your food 5. Getting in or out of bed 6. Using the toilet, including getting up or down. The responses to all 5 questions by a person without disability was "no problems". The percentage with no difficulty taking medications was determined by asking the same question in sub-question 11. Taking medications. The number of people, expressed in percent, with no difficulty walking a short distance was determined based on a no response marked for the question: We need to understand difficulties people may have with various activities because of a health or physical problem. Please tell me whether you have any difficulty doing each of the everyday activities on card 10. Exclude any difficulties that you expect to last less than three months. 1. Walking 100 metres. The life expectancy in the original methodology was replaced with healthylife years which better corresponded with the relevant dimension. For this indicator, the data were taken from the Eurostat database. The percentage not obese was determined based on the respondent's information about weight (Approximately how much do you weigh?) and height (How tall are you?) (in cases where no information about the height was provided, we used the available data from the previous SHARE). An individual with a BMl above 30 was considered obese.

The dimension of social commitment was defined by 2 variables - percentage participating in an economic or social activity (socially connected), and percentage in contact with at least one child. As for the percentage of persons participating in economic or social activities, at least one YES answer to the following questions was decisive: Which of the activities listed on this card - if any - have you done in the past twelve months? 1. Done voluntary or charity work 5. Gone to a sport, social or other kind of club 6. Taken part in activities of a religious organization (church, synagogue, mosque etc.) 7. Taken part in a political or community-related organization or response 2: Employed or selfemployed: In general, which of the following best describes your current employment situation? Percentage in contact with at least one child was determined based on the question: During the past twelve months, how often did you have contact with [name of child], personally, by phone or mail?. In successive steps, the survey asked questions about all living children. We considered the following answers satisfactory: 1. Daily 2. Several times a week 3. About once a week 4. About every two weeks 5. About once a month 6. Less than once a month.

The emotional well-being dimension had 3 variables - percentage with no report of depression (a non-clinical measure), the suicide rate for older adults (reverse coded), and percentage thriving (satisfied with current life and future prospects). The percentage with no report of depression was defined based on subjective feelings: In the last month, have you been sad or depressed? The suicide rate for adults was taken from the data of the World Health Organization. The number of persons, expressed in percent who are thriving, required feeling satisfied in the present and expecting a positive future. A rating of 7 or higher to the following question was required: On a scale from 0 to 10 where 0 means completely dissatisfied and 10 means completely satisfied, how satisfied are you with your life? and the response Often to the question How often do you feel that the future looks good for you? (Often, sometimes, rarely or never?). 
Subsequently, a synthesized quality of life index was calculated in three steps: determining the score of the indicators (Tab. 1), calculating the dimension score, and calculating the final summary index. For individual indicators, the score was determined by the best practice method. This means that the best value was identified and $100 \%$ was allocated to each variable. The values for other countries were calculated as the proportion of the percent value and the above base and could range from $0 \%$ to $100 \%$ of the value. The average dimension score was calculated from individual indicators expressed as a percentage (Tab. 2).

The aggregate quality of life index was calculated as a weighted arithmetic average of the scores on individual indicators. An analysis of the structure of a correlation matrix (Tab. 3) was used to calculate the weight of individual indicators.

The calculation of the overall score of quality of life was carried out as follows:

$\mathrm{QL}=\left(5,88 \mathrm{x}_{1}+5,90 \mathrm{x}_{2}+5,23 \mathrm{x}_{3}+2,65 \mathrm{x}_{4}+\right.$ $+5,87 x_{5}+5,08 x_{6}+5,66 x_{7}+5,92 x_{8}+1,48 x_{9}+$ $\left.+3,86 \mathrm{x}_{10}+2,41 \mathrm{x}_{11}+6,42 \mathrm{x}_{12}\right) / 56,37$

In this case, weights gained by analysing the structure of the correlation matrix have high values in indicators that are more correlated with the greatest number of other variables possible and that depend on the intensity of this correlation as well as on the number of strongly correlated indicators. These weights enable us to determine the significance of each indicator while the "clearer" the type of indicator, the higher the value. We applied the sums of pair coefficients in correlations as weights of indicators that we eventually divided by the total sum of pair coefficients. This ensured that the total quality of life index also reached values of 0-100\%. Thus, to obtain a $100 \%$ quality of life index a country would need to achieve the best value for each of the 12 variables.

However, we must point out some limitations of the study. First, we had to drop the aim of evaluating quality of life in several age categories of seniors. Age is one of the determining factors of quality of life and such approach would enable further longitudinal analysis. However, the numbers of responses in this case would be too low and the information would be distorted. Second, when it comes to quality of life, it would be interesting to examine not only the quantity of some indicators (family relations), but also their quality, or the satisfaction of a senior with a given indicator. Nevertheless, we are convinced that this study offers a comparable and multidimensional image of the quality of life of seniors in various countries of Europe.

\section{Characteristics of the group of respondents}

In accordance with the conclusions of other studies, we found it necessary to characterise the sample in terms of elementary demographic and socioeconomic factors. However, these independent variables (since they do not fall into any of the domains) had to be defined as these factors also affected the respondents' preferences.

A total of 29,679 respondents older than 65 participated in the survey (Tab. 4). The femininity index was $1259 \%$ as there were 13,138 men and 16,541 women. Estonia had the largest number of respondents (2291) while Poland had the smallest sample (398 men). The average age of respondents in individual countries ranged from 72.60 in Portugal (men) to 76.04 in Spain (women). Significant characteristics affecting the quality of life also included the respondent's marital status. In this respect, a difference needs to be pointed out in the marital status of men and women. Married people dominated in both groups. Nevertheless, the number of married men was higher, and the difference was much more significant in Eastern Europe than in Western Europe. This fact results from different life expectancy values and particularly from the bigger gap between men and women (the so-called gender gap) in Eastern Europe. The smallest percentage of married respondents was from Austria (42.48\%, women) and the highest 
Table 1. Values of indicators of quality of life

\begin{tabular}{|c|c|c|c|c|c|c|}
\hline \multirow[b]{2}{*}{ Country } & \multirow[b]{2}{*}{ Gender } & \multicolumn{2}{|c|}{$\begin{array}{c}\text { Material } \\
\text { dimension }\end{array}$} & \multicolumn{3}{|c|}{$\begin{array}{c}\text { Health } \\
\text { dimension }\end{array}$} \\
\hline & & $\begin{array}{l}\text { median } \\
\text { income }\end{array}$ & $\begin{array}{c}\text { not } \\
\text { in absolute } \\
\text { poverty }\end{array}$ & $\begin{array}{c}\text { no } \\
\text { disability }\end{array}$ & $\begin{array}{c}\text { no } \\
\text { difficulty } \\
\text { taking meds }\end{array}$ & $\begin{array}{c}\text { no } \\
\text { functional } \\
\text { limitations }\end{array}$ \\
\hline Sweden & $\begin{array}{l}\text { men } \\
\text { women }\end{array}$ & $\begin{array}{l}16,838 \\
16,164\end{array}$ & $\begin{array}{l}93.73 \\
85.12\end{array}$ & $\begin{array}{l}86.14 \\
86.49\end{array}$ & $\begin{array}{l}95.79 \\
96.26\end{array}$ & $\begin{array}{l}90.33 \\
88.54\end{array}$ \\
\hline Denmark & $\begin{array}{l}\text { men } \\
\text { women }\end{array}$ & $\begin{array}{l}14,612 \\
14,612\end{array}$ & $\begin{array}{l}89.19 \\
81.78\end{array}$ & $\begin{array}{l}88.66 \\
88.69\end{array}$ & $\begin{array}{l}95.46 \\
96.59\end{array}$ & $\begin{array}{l}92.05 \\
87.61\end{array}$ \\
\hline Netherlands & $\begin{array}{l}\text { men } \\
\text { women }\end{array}$ & $\begin{array}{l}17,561 \\
18,293\end{array}$ & $\begin{array}{l}97.55 \\
97.31\end{array}$ & $\begin{array}{l}92.10 \\
89.71\end{array}$ & $\begin{array}{l}98.18 \\
98.49\end{array}$ & $\begin{array}{l}90.12 \\
87.79\end{array}$ \\
\hline Belgium & $\begin{array}{l}\text { men } \\
\text { women }\end{array}$ & $\begin{array}{l}15,349 \\
15,349\end{array}$ & $\begin{array}{l}90.78 \\
89.43\end{array}$ & $\begin{array}{l}80.71 \\
73.44\end{array}$ & $\begin{array}{r}97.13 \\
95.43\end{array}$ & $\begin{array}{l}85.33 \\
76.86\end{array}$ \\
\hline France & $\begin{array}{l}\text { men } \\
\text { women }\end{array}$ & $\begin{array}{l}16,235 \\
15,529\end{array}$ & $\begin{array}{r}87.71 \\
80.32\end{array}$ & $\begin{array}{l}84.08 \\
82.35\end{array}$ & $\begin{array}{l}97.35 \\
96.56\end{array}$ & $\begin{array}{l}86.64 \\
80.80\end{array}$ \\
\hline Switzerland & $\begin{array}{l}\text { men } \\
\text { women }\end{array}$ & $\begin{array}{l}22,557 \\
21,429\end{array}$ & $\begin{array}{l}94.27 \\
92.42\end{array}$ & $\begin{array}{l}92.50 \\
89.54\end{array}$ & $\begin{array}{l}99.41 \\
98.54\end{array}$ & $\begin{array}{l}93.67 \\
92.57\end{array}$ \\
\hline Germany & $\begin{array}{l}\text { men } \\
\text { women }\end{array}$ & $\begin{array}{l}16,709 \\
16,709\end{array}$ & $\begin{array}{r}94.33 \\
89.12\end{array}$ & $\begin{array}{l}84.65 \\
80.94\end{array}$ & $\begin{array}{l}96.97 \\
95.72\end{array}$ & $\begin{array}{l}85.45 \\
80.51\end{array}$ \\
\hline Austria & $\begin{array}{l}\text { men } \\
\text { women }\end{array}$ & $\begin{array}{l}16,829 \\
16,098\end{array}$ & $\begin{array}{l}94.21 \\
82.12\end{array}$ & $\begin{array}{l}86.56 \\
84.28\end{array}$ & $\begin{array}{l}97.35 \\
97.88\end{array}$ & $\begin{array}{l}90.68 \\
85.88\end{array}$ \\
\hline Slovenia & $\begin{array}{l}\text { men } \\
\text { women }\end{array}$ & $\begin{array}{r}10,476 \\
9524\end{array}$ & $\begin{array}{l}62.73 \\
52.61\end{array}$ & $\begin{array}{l}84.51 \\
86.97\end{array}$ & $\begin{array}{l}97.13 \\
97.56\end{array}$ & $\begin{array}{l}81.84 \\
75.71\end{array}$ \\
\hline Italy & $\begin{array}{l}\text { men } \\
\text { women }\end{array}$ & $\begin{array}{r}11,111 \\
11,383\end{array}$ & $\begin{array}{l}74.55 \\
68.17\end{array}$ & $\begin{array}{l}87.71 \\
79.21\end{array}$ & $\begin{array}{l}95.52 \\
95.27\end{array}$ & $\begin{array}{l}84.90 \\
72.22\end{array}$ \\
\hline Spain & $\begin{array}{l}\text { men } \\
\text { women }\end{array}$ & $\begin{array}{l}8919 \\
9405\end{array}$ & $\begin{array}{r}53.5 \\
49.75\end{array}$ & $\begin{array}{l}83.81 \\
74.59\end{array}$ & $\begin{array}{l}89.31 \\
90.16\end{array}$ & $\begin{array}{l}82.96 \\
69.61\end{array}$ \\
\hline Portugal & $\begin{array}{l}\text { men } \\
\text { women }\end{array}$ & $\begin{array}{l}8113 \\
8451\end{array}$ & $\begin{array}{r}48.8 \\
46.08\end{array}$ & $\begin{array}{l}83.45 \\
70.41\end{array}$ & $\begin{array}{l}98.88 \\
93.82\end{array}$ & $\begin{array}{l}80.54 \\
67.35\end{array}$ \\
\hline Czech Republic & $\begin{array}{l}\text { men } \\
\text { women }\end{array}$ & $\begin{array}{l}8568 \\
8291\end{array}$ & $\begin{array}{l}48.72 \\
29.36\end{array}$ & $\begin{array}{l}87.61 \\
84.95\end{array}$ & $\begin{array}{l}97.49 \\
97.37\end{array}$ & $\begin{array}{l}88.16 \\
82.97\end{array}$ \\
\hline Poland & $\begin{array}{l}\text { men } \\
\text { women }\end{array}$ & $\begin{array}{l}6672 \\
6196\end{array}$ & $\begin{array}{l}33.24 \\
27.63\end{array}$ & $\begin{array}{l}80.05 \\
71.71\end{array}$ & $\begin{array}{l}94.70 \\
93.20\end{array}$ & $\begin{array}{l}79.04 \\
75.88\end{array}$ \\
\hline Hungary & $\begin{array}{l}\text { men } \\
\text { women }\end{array}$ & $\begin{array}{l}6638 \\
6804\end{array}$ & $\begin{array}{l}22.36 \\
16.97\end{array}$ & $\begin{array}{l}81.57 \\
81.70\end{array}$ & $\begin{array}{l}96.93 \\
96.95\end{array}$ & $\begin{array}{l}71.50 \\
60.74\end{array}$ \\
\hline Estonia & $\begin{array}{l}\text { men } \\
\text { women }\end{array}$ & $\begin{array}{l}6587 \\
6455\end{array}$ & $\begin{array}{l}24.02 \\
16.34\end{array}$ & $\begin{array}{r}79.10 \\
77.16\end{array}$ & $\begin{array}{l}95.86 \\
97.24\end{array}$ & $\begin{array}{l}80.52 \\
77.99\end{array}$ \\
\hline Minimum & & 6196 & 16.34 & 70.41 & 89.31 & 60.74 \\
\hline Maximum & & 22,557 & 97.55 & 92.50 & 99.41 & 93.67 \\
\hline Mean & & 12,640 & 66.07 & 83.29 & 96.27 & 82.09 \\
\hline Variance & & $22,075,957$ & 741.55 & 30.09 & 4.72 & 60.54 \\
\hline
\end{tabular}

Source: based on Share 4, Release 1.1.1, Eurostat (2014), WHO (2014). 


\begin{tabular}{|c|c|c|c|c|c|c|}
\hline \multicolumn{2}{|c|}{$\begin{array}{c}\text { Health } \\
\text { dimension }\end{array}$} & \multicolumn{2}{|c|}{$\begin{array}{c}\text { Social } \\
\text { dimension }\end{array}$} & \multicolumn{3}{|c|}{$\begin{array}{l}\text { Emotional } \\
\text { dimension }\end{array}$} \\
\hline $\begin{array}{l}\text { healthy } \\
\text { years }\end{array}$ & $\begin{array}{c}\text { not } \\
\text { obese }\end{array}$ & $\begin{array}{l}\text { socially } \\
\text { connected }\end{array}$ & $\begin{array}{c}\text { contact } \\
\text { with children }\end{array}$ & $\begin{array}{c}\text { not } \\
\text { depressed }\end{array}$ & $\begin{array}{l}\text { suicide } \\
\text { rate } \\
\text { (reverse) }\end{array}$ & thriving \\
\hline 13.90 & 84.08 & 56.15 & 92.03 & 75.20 & -27.23 & 41.69 \\
\hline 15.20 & 83.90 & 53.72 & 89.97 & 63.53 & -8.47 & 41.30 \\
\hline 12.40 & 88.35 & 66.81 & 89.28 & 78.45 & -37.47 & 53.66 \\
\hline 13.00 & 86.06 & 67.35 & 90.31 & 65.88 & -11.28 & 50.00 \\
\hline 10.40 & 84.37 & 66.04 & 92.38 & 79.66 & -16.09 & 56.60 \\
\hline 9.90 & 83.21 & 63.34 & 90.53 & 61.60 & -6.94 & 53.24 \\
\hline 9.80 & 81.85 & 52.16 & 86.70 & 71.73 & -41.69 & 38.89 \\
\hline 10.30 & 80.54 & 45.98 & 86.30 & 52.13 & -13.28 & 33.92 \\
\hline 9.70 & 80.32 & 46.99 & 84.41 & 62.69 & -47.53 & 29.45 \\
\hline 9.90 & 82.38 & 43.23 & 87.19 & 43.94 & -12.41 & 25.05 \\
\hline 12.70 & 85.25 & 63.40 & 85.90 & 70.51 & -48.25 & 59.41 \\
\hline 12.80 & 86.62 & 52.20 & 85.24 & 53.73 & -24.57 & 54.58 \\
\hline 6.70 & 82.05 & 52.99 & 87.37 & 66.80 & -33.10 & 41.08 \\
\hline 7.30 & 79.42 & 47.79 & 87.90 & 42.17 & -10.76 & 31.36 \\
\hline 8.30 & 81.89 & 52.79 & 80.93 & 73.72 & -49.12 & 44.79 \\
\hline 8.30 & 81.19 & 43.69 & 84.80 & 56.93 & -12.34 & 37.25 \\
\hline 6.20 & 76.80 & 39.19 & 96.18 & 69.77 & -58.52 & 32.35 \\
\hline 6.90 & 77.46 & 38.03 & 88.60 & 55.60 & -20.29 & 31.56 \\
\hline 8.10 & 83.01 & 29.63 & 90.19 & 70.37 & -19.40 & 24.13 \\
\hline 7.00 & 80.45 & 30.03 & 88.57 & 47.90 & -4.45 & 19.82 \\
\hline 9.70 & 77.78 & 26.05 & 88.98 & 71.71 & -24.49 & 19.02 \\
\hline 9.30 & 71.05 & 28.42 & 88.88 & 43.36 & -5.99 & 13.15 \\
\hline 7.80 & 83.29 & 24.20 & 89.11 & 62.73 & -39.95 & 12.01 \\
\hline 6.30 & 75.87 & 26.29 & 82.90 & 37.86 & -9.08 & 8.63 \\
\hline 8.40 & 75.20 & 36.17 & 91.88 & 69.89 & -35.12 & 15.92 \\
\hline 8.70 & 71.18 & 28.94 & 90.23 & 51.52 & -7.11 & 13.79 \\
\hline 7.60 & 73.70 & 41.22 & 86.18 & 54.71 & -32.14 & 21.49 \\
\hline 8.30 & 68.15 & 50.77 & 95.41 & 40.22 & -5.86 & 17.36 \\
\hline 6.00 & 75.57 & 23.55 & 82.05 & 68.36 & -74.06 & 15.64 \\
\hline 6.00 & 73.70 & 25.00 & 81.73 & 52.21 & -17.33 & 11.74 \\
\hline 5.60 & 78.02 & 27.46 & 88.06 & 60.80 & -44.00 & 12.82 \\
\hline 5.70 & 68.17 & 27.49 & 82.86 & 40.97 & -10.53 & 12.90 \\
\hline 5.60 & 68.15 & 23.55 & 80.93 & 37.86 & -74.06 & 8.63 \\
\hline 15.20 & 88.35 & 67.35 & 96.18 & 79.66 & -4.45 & 59.41 \\
\hline 9.01 & 79.40 & 43.03 & 87.91 & 59.90 & -25.28 & 30.46 \\
\hline 6.36 & 27.18 & 194.74 & 13.25 & 142.07 & 311.62 & 237.53 \\
\hline
\end{tabular}


Table 2. Domains and aggregate index of quality of life

\begin{tabular}{|c|c|c|c|c|c|c|c|c|}
\hline \multirow{3}{*}{ Country } & \multirow{3}{*}{ Gender } & \multicolumn{3}{|c|}{$\begin{array}{c}\text { Material } \\
\text { dimension }\end{array}$} & \multicolumn{4}{|c|}{$\begin{array}{c}\text { Health } \\
\text { dimension }\end{array}$} \\
\hline & & \multirow[b]{2}{*}{ domain } & \multicolumn{2}{|c|}{ indicator } & \multirow[b]{2}{*}{ domain } & \multicolumn{3}{|c|}{ indicator } \\
\hline & & & $\begin{array}{l}\text { median } \\
\text { income }\end{array}$ & $\begin{array}{c}\text { not } \\
\text { in absolute } \\
\text { poverty }\end{array}$ & & $\begin{array}{c}\text { no } \\
\text { disability }\end{array}$ & $\begin{array}{c}\text { no } \\
\text { difficulty } \\
\text { taking } \\
\text { meds }\end{array}$ & $\begin{array}{c}\text { no } \\
\text { functional } \\
\text { limitations }\end{array}$ \\
\hline \multirow[t]{2}{*}{ Sweden } & men & 85 & 75 & 96 & 95 & 93 & 96 & 96 \\
\hline & women & 79 & 72 & 87 & 96 & 94 & 97 & 95 \\
\hline \multirow[t]{2}{*}{ Denmark } & men & 78 & 65 & 91 & 94 & 96 & 96 & 98 \\
\hline & women & 74 & 65 & 84 & 94 & 96 & 97 & 94 \\
\hline \multirow[t]{2}{*}{ Netherlands } & men & 89 & 78 & 100 & 92 & 100 & 99 & 96 \\
\hline & women & 90 & 81 & 100 & 90 & 97 & 99 & 94 \\
\hline \multirow[t]{2}{*}{ Belgium } & men & 81 & 68 & 93 & 87 & 87 & 98 & 91 \\
\hline & women & 80 & 68 & 92 & 83 & 79 & 96 & 82 \\
\hline \multirow[t]{2}{*}{ France } & men & 81 & 72 & 90 & 87 & 91 & 98 & 92 \\
\hline & women & 76 & 69 & 82 & 86 & 89 & 97 & 86 \\
\hline \multirow[t]{2}{*}{ Switzerland } & men & 98 & 100 & 97 & 96 & 100 & 100 & 100 \\
\hline & women & 95 & 95 & 95 & 95 & 97 & 99 & 99 \\
\hline \multirow[t]{2}{*}{ Germany } & men & 85 & 74 & 97 & 83 & 92 & 98 & 91 \\
\hline & women & 83 & 74 & 91 & 82 & 88 & 96 & 86 \\
\hline \multirow[t]{2}{*}{ Austria } & men & 86 & 75 & 97 & 87 & 94 & 98 & 97 \\
\hline & women & 78 & 71 & 84 & 86 & 91 & 98 & 92 \\
\hline \multirow[t]{2}{*}{ Slovenia } & men & 55 & 46 & 64 & 81 & 91 & 98 & 87 \\
\hline & women & 48 & 42 & 54 & 81 & 94 & 98 & 81 \\
\hline \multirow[t]{2}{*}{ Italy } & men & 63 & 49 & 76 & 86 & 95 & 96 & 91 \\
\hline & women & 60 & 50 & 70 & 79 & 86 & 96 & 77 \\
\hline \multirow[t]{2}{*}{ Spain } & men & 47 & 40 & 55 & 84 & 91 & 90 & 89 \\
\hline & women & 46 & 42 & 51 & 77 & 81 & 91 & 74 \\
\hline \multirow[t]{2}{*}{ Portugal } & men & 43 & 36 & 50 & 84 & 90 & 99 & 86 \\
\hline & women & 42 & 37 & 47 & 74 & 76 & 94 & 72 \\
\hline \multirow[t]{2}{*}{ Czech Republic } & men & 44 & 38 & 50 & 85 & 95 & 98 & 94 \\
\hline & women & 33 & 37 & 30 & 83 & 92 & 98 & 89 \\
\hline \multirow[t]{2}{*}{ Poland } & men & 32 & 30 & 34 & 80 & 87 & 95 & 84 \\
\hline & women & 28 & 27 & 28 & 77 & 78 & 94 & 81 \\
\hline \multirow[t]{2}{*}{ Hungary } & men & 26 & 29 & 23 & 77 & 88 & 97 & 76 \\
\hline & women & 24 & 30 & 17 & 75 & 88 & 98 & 65 \\
\hline \multirow[t]{2}{*}{ Estonia } & men & 27 & 29 & 25 & 79 & 86 & 96 & 86 \\
\hline & women & 23 & 29 & 17 & 76 & 83 & 98 & 83 \\
\hline
\end{tabular}

Source: based on Share 4, Release 1.1.1, Eurostat (2014), WHO (2014). 


\begin{tabular}{|c|c|c|c|c|c|c|c|c|c|}
\hline \multirow{2}{*}{\multicolumn{2}{|c|}{$\begin{array}{c}\text { Health } \\
\text { dimension }\end{array}$}} & \multicolumn{3}{|c|}{$\begin{array}{c}\text { Social } \\
\text { dimension }\end{array}$} & \multicolumn{4}{|c|}{$\begin{array}{l}\text { Emotional } \\
\text { dimension }\end{array}$} & \multirow{3}{*}{$\begin{array}{l}\text { Aggregate } \\
\text { index }\end{array}$} \\
\hline & & \multirow[b]{2}{*}{ domain } & \multicolumn{2}{|c|}{ indicator } & \multirow[b]{2}{*}{ domain } & \multicolumn{3}{|c|}{ indicator } & \\
\hline $\begin{array}{l}\text { healthy } \\
\text { years }\end{array}$ & $\begin{array}{c}\text { not } \\
\text { obese }\end{array}$ & & $\begin{array}{c}\text { socially } \\
\text { connected }\end{array}$ & $\begin{array}{l}\text { contact } \\
\text { with } \\
\text { children }\end{array}$ & & $\begin{array}{c}\text { not } \\
\text { depressed }\end{array}$ & $\begin{array}{l}\text { suicide } \\
\text { rate } \\
\text { (reverse) }\end{array}$ & thriving & \\
\hline 91 & 95 & 90 & 83 & 96 & 60 & 94 & 16 & 70 & 85 \\
\hline 100 & 95 & 87 & 80 & 94 & 67 & 80 & 52 & 70 & 85 \\
\hline 82 & 100 & 96 & 99 & 93 & 67 & 98 & 12 & 90 & 88 \\
\hline 86 & 97 & 97 & 100 & 94 & 69 & 83 & 39 & 84 & 86 \\
\hline 68 & 95 & 97 & 98 & 96 & 74 & 100 & 28 & 95 & 90 \\
\hline 65 & 94 & 94 & 94 & 94 & 77 & 77 & 64 & 90 & 88 \\
\hline 64 & 93 & 84 & 77 & 90 & 55 & 90 & 11 & 65 & 79 \\
\hline 68 & 91 & 79 & 68 & 90 & 52 & 65 & 33 & 57 & 74 \\
\hline 64 & 91 & 79 & 70 & 88 & 46 & 79 & 9 & 50 & 76 \\
\hline 65 & 93 & 77 & 64 & 91 & 44 & 55 & 36 & 42 & 72 \\
\hline 84 & 96 & 92 & 94 & 89 & 66 & 89 & 9 & 100 & 92 \\
\hline 84 & 98 & 83 & 78 & 89 & 59 & 67 & 18 & 92 & 88 \\
\hline 44 & 93 & 85 & 79 & 91 & 55 & 84 & 13 & 69 & 79 \\
\hline 48 & 90 & 81 & 71 & 91 & 49 & 53 & 41 & 53 & 74 \\
\hline 55 & 93 & 81 & 78 & 84 & 59 & 93 & 9 & 75 & 81 \\
\hline 55 & 92 & 77 & 65 & 88 & 57 & 71 & 36 & 63 & 76 \\
\hline 41 & 87 & 79 & 58 & 100 & 50 & 88 & 8 & 54 & 67 \\
\hline 45 & 88 & 74 & 56 & 92 & 48 & 70 & 22 & 53 & 65 \\
\hline 53 & 94 & 69 & 44 & 94 & 51 & 88 & 23 & 41 & 69 \\
\hline 46 & 91 & 68 & 45 & 92 & 64 & 60 & 100 & 33 & 66 \\
\hline 64 & 88 & 66 & 39 & 93 & 47 & 90 & 18 & 32 & 63 \\
\hline 61 & 80 & 67 & 42 & 92 & 50 & 54 & 74 & 22 & 59 \\
\hline 51 & 94 & 64 & 36 & 93 & 37 & 79 & 11 & 20 & 59 \\
\hline 41 & 86 & 63 & 39 & 86 & 37 & 48 & 49 & 15 & 54 \\
\hline 55 & 85 & 75 & 54 & 96 & 42 & 88 & 13 & 27 & 64 \\
\hline 57 & 81 & 68 & 43 & 94 & 50 & 65 & 63 & 23 & 59 \\
\hline 50 & 83 & 75 & 61 & 90 & 40 & 69 & 14 & 36 & 59 \\
\hline 55 & 77 & 87 & 75 & 99 & 52 & 50 & 76 & 29 & 59 \\
\hline 39 & 86 & 60 & 35 & 85 & 39 & 86 & 6 & 26 & 53 \\
\hline 39 & 83 & 61 & 37 & 85 & 37 & 66 & 26 & 20 & 50 \\
\hline 37 & 88 & 66 & 41 & 92 & 36 & 76 & 10 & 22 & 54 \\
\hline 38 & 77 & 63 & 41 & 86 & 38 & 51 & 42 & 22 & 51 \\
\hline
\end{tabular}

Source: based on Share 4, Release 1.1.1, Eurostat (2014), WHO (2014). 
Table 3. Correlation matrix of factors

\begin{tabular}{|c|c|c|c|c|c|c|c|c|c|c|c|c|}
\hline & 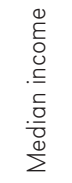 & 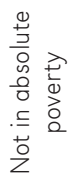 & 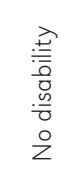 & 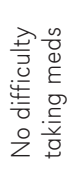 & 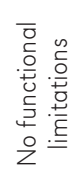 & 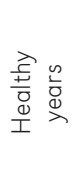 & 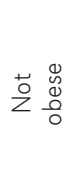 & 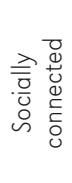 & 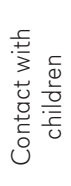 & 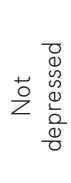 & 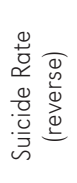 & : \\
\hline Median income & $x$ & 0.93 & 0.56 & 0.41 & 0.70 & 0.66 & 0.78 & 0.78 & -0.03 & 0.34 & -0.13 & 0.88 \\
\hline $\begin{array}{l}\text { Not in absolute } \\
\text { poverty }\end{array}$ & 0.93 & $x$ & 0.52 & 0.27 & 0.69 & 0.63 & 0.83 & 0.77 & 0.11 & 0.43 & -0.13 & 0.84 \\
\hline No disability & 0.56 & 0.52 & $x$ & 0.56 & 0.74 & 0.48 & 0.65 & 0.52 & 0.18 & 0.72 & -0.40 & 0.69 \\
\hline $\begin{array}{l}\text { No difficulty } \\
\text { taking meds }\end{array}$ & 0.41 & 0.27 & 0.56 & $x$ & 0.40 & 0.06 & 0.39 & 0.33 & -0.14 & 0.27 & -0.33 & 0.43 \\
\hline $\begin{array}{l}\text { No functional } \\
\text { limitations }\end{array}$ & 0.70 & 0.69 & 0.74 & 0.40 & $x$ & 0.65 & 0.67 & 0.71 & 0.27 & 0.65 & -0.37 & 0.76 \\
\hline Healthy years & 0.66 & 0.63 & 0.48 & 0.06 & 0.65 & $x$ & 0.63 & 0.69 & 0.24 & 0.37 & -0.01 & 0.68 \\
\hline Not obese & 0.78 & 0.83 & 0.65 & 0.39 & 0.67 & 0.63 & $x$ & 0.63 & 0.04 & 0.58 & -0.32 & 0.77 \\
\hline $\begin{array}{l}\text { Socially } \\
\text { connected }\end{array}$ & 0.78 & 0.77 & 0.52 & 0.33 & 0.71 & 0.69 & 0.63 & $x$ & 0.25 & 0.41 & -0.09 & 0.91 \\
\hline $\begin{array}{l}\text { Contact with } \\
\text { children }\end{array}$ & -0.03 & 0.11 & 0.18 & -0.14 & 0.27 & 0.24 & 0.04 & 0.25 & $x$ & 0.21 & 0.22 & 0.13 \\
\hline Not depressed & 0.34 & 0.43 & 0.72 & 0.27 & 0.65 & 0.37 & 0.58 & 0.41 & 0.21 & $x$ & -0.63 & 0.54 \\
\hline $\begin{array}{l}\text { Suicide Rate } \\
\text { (reverse) }\end{array}$ & -0.13 & -0.13 & -0.40 & -0.33 & -0.37 & -0.01 & -0.32 & -0.09 & 0.22 & -0.63 & $x$ & -0.22 \\
\hline Thriving & 0.88 & 0.84 & 0.69 & 0.43 & 0.76 & 0.68 & 0.77 & 0.91 & 0.13 & 0.54 & -0.22 & $x$ \\
\hline$\Sigma$ & 5.88 & 5.90 & 5.23 & 2.65 & 5.87 & 5.08 & 5.66 & 5.92 & 1.48 & 3.86 & 2.41 & 6.42 \\
\hline
\end{tabular}

percentage of married respondents was from Italy (85.49\%, men). Registered partnerships, except for Sweden (6.29 or $8.24 \%$ ), represented negligible values. The same situation existed for married but separated individuals. The percentage of singles was $1-6 \%$ and divorced $0.5-11.7 \%$, with the more significant values in Northern Europe and the Czech Republic. Those who were widowed ranged from 6.94\% (Italy - men) to 44.61\% (Hungary - women). Significant differences between individual countries were also identified in terms of education. Southern European countries, together with Poland and France, had a high rate of people with an elementary education (40$60 \%$ ). Education was classified in accordance with the international standard classification of education (ISCED). Eastern European countries traditionally had a high percentage of lower secondary education or second stage of elementary education. Western and Northern European countries had had a higher level of higher secondary or the first stage or tertiary education. Post-secondary, non-tertiary education reached more significant values in Estonia and the Czech Republic (10-15\%).

\section{Dimensions of quality of life}

\section{Material dimension}

When growing older, a person goes through a number of transitions. Receiving an old-age pension is an extremely significant transition in terms of the psychological issues (loss of social roles, social contacts, being perceived as a senior by others...) and mental issues. One's income usually decreases (the 
transition from income from employment to pension), which implies living in smaller households (not only because children will have moved away but also because, at some stage, a spouse dies); frequently, flats in large cities are left to the children or people move back to live with their now adult children due to the high costs of keeping a separate household.

Thus, it is evident that the amount of a pension plays a key role in the evaluation of the material dimension of seniors. In European countries, the score for this dimension significantly varied from 72 points (for men) and 74 points (for women). Among all dimensions evaluated, this showed the highest range of values among countries. The highest level of satisfaction in men above 65 years was reported in Switzerland, the Netherlands and Austria, and the best female ratings of satisfaction were in Switzerland and the Netherlands (Fig. 1a). On the other side of the spectrum, respondents of both genders living in Estonia, Hungary and Poland had the lowest quality of life in terms of material aspects. The differences between the countries raise hypothetical as well as actual questions about the care for seniors and the social systems of individual countries. A general difference in terms of location was identified: prosperous Western and Northern European seniors were more prosperous than Southern and Eastern European seniors.

This was due to two main factors. National GDP was a major factor explaining the variances between European countries. The correlation coefficient between national GDP and the economic dimension was 0.916 and was statistically significant at the level of $\alpha=5 \%$. Thus, when looking at Europe in terms of nations, the material dimension of seniors significantly corresponded with the economic development of the countries. The pension systems in individual countries represented the second significant factor. To date, Eastern European countries almost exclusively pay pensions from the state sector, while in Sweden, Denmark and the Netherlands, non-public, occupation-related pension expenditures today account for 2 to $5 \%$ of GDP(European Commission, 2009). The report of the Melbourne Mercer Global Pensions Index 2012 also rated the Danish, Dutch, Australian, Swedish and Swiss pension systems as the best. The Danish pension system is composed of the basic public pension pillar, a fully-funded contribution system, and a statutory occupation-based system. Similarly, other pension systems in Northern and Western Europe have more than one component, frequently supported by favourable tax regulations. As a result, these countries have a high income-replacement ratio and people retiring and beginning to receive a pension experience a much less radical drop in their standard of living. However, this factor is also influenced by the personal approach of individuals. Participation in voluntary pension security funds in the countries of Eastern and Southern Europe is very low.

Higher values for the partial index of the material dimension were found among men (except in the Netherlands). This may relate to the higher level of education of the men born around the 1950s, a fact that was reflected in our sample of respondents (Tab. 4). There was a slightly linear relationship for both genders between the score of the material dimension and the number of years spent in acquiring education. Moreover, men remained in the labour market longer than women, which may have contributed to their higher income. The marital status of women (widowed more frequently), fewer years in the labour market (maternity leave) and lower education in the monitored generations of seniors are factors that limited the income or risk of poverty of women. After segmentation into Western and Northern, and Southern and Eastern Europe, the number of married individuals from both groups of countries showed a linear relationship between the material dimension and marital status, in which $\alpha=5 \%$ can be considered as statistically-significant. Nevertheless, this conclusion is limited by the smaller number of statistical units after segmentation. This fact was also validated by Poleij and Kraan 
Table 4. The socio-demographic data of participants

\begin{tabular}{|c|c|c|c|c|c|c|c|c|}
\hline \multirow{2}{*}{ Country } & \multirow{2}{*}{ Sex } & \multirow{2}{*}{$\begin{array}{c}\text { Respon- } \\
\text { dents }\end{array}$} & \multirow{2}{*}{ Mean age } & \multicolumn{5}{|c|}{ Marital status (in \%) } \\
\hline & & & & $M-L T$ & $\mathrm{RP}$ & M-LS & $S$ & $\mathrm{D}$ \\
\hline Austria & $\begin{array}{l}\text { M } \\
W\end{array}$ & $\begin{array}{l}1110 \\
1516\end{array}$ & $\begin{array}{l}73.42 \\
74.03\end{array}$ & $\begin{array}{l}75.32 \\
42.48\end{array}$ & $\begin{array}{l}0.36 \\
0.20\end{array}$ & $\begin{array}{l}1.80 \\
1.39\end{array}$ & $\begin{array}{l}4.95 \\
6.13\end{array}$ & $\begin{array}{r}6.49 \\
10.95\end{array}$ \\
\hline Germany & $\begin{array}{l}\text { M } \\
W\end{array}$ & $\begin{array}{l}495 \\
467\end{array}$ & $\begin{array}{r}73.57 \\
74.13\end{array}$ & $\begin{array}{l}81.41 \\
64.88\end{array}$ & $\begin{array}{l}0.00 \\
0.43\end{array}$ & $\begin{array}{l}0.40 \\
1.28\end{array}$ & $\begin{array}{l}3.84 \\
2.57\end{array}$ & $\begin{array}{l}3.03 \\
5.35\end{array}$ \\
\hline Sweden & $\begin{array}{l}M \\
W\end{array}$ & $\begin{array}{l}643 \\
699\end{array}$ & $\begin{array}{l}74.31 \\
74.23\end{array}$ & $\begin{array}{l}71.70 \\
59.23\end{array}$ & $\begin{array}{l}8.24 \\
6.29\end{array}$ & $\begin{array}{l}0.31 \\
0.14\end{array}$ & $\begin{array}{l}3.27 \\
3.58\end{array}$ & $\begin{array}{l}5.13 \\
9.01\end{array}$ \\
\hline Nether-land & $\begin{array}{l}M \\
W\end{array}$ & $\begin{array}{l}659 \\
730\end{array}$ & $\begin{array}{l}73.39 \\
74.00\end{array}$ & $\begin{array}{l}78.00 \\
62.05\end{array}$ & $\begin{array}{l}1.82 \\
1.64\end{array}$ & $\begin{array}{l}0.91 \\
0.82\end{array}$ & $\begin{array}{l}2.73 \\
2.60\end{array}$ & $\begin{array}{l}3.34 \\
5.62\end{array}$ \\
\hline Spain & $\begin{array}{l}\mathrm{M} \\
\mathrm{W}\end{array}$ & $\begin{array}{r}953 \\
1104\end{array}$ & $\begin{array}{l}75.16 \\
76.04\end{array}$ & $\begin{array}{l}82.16 \\
61.50\end{array}$ & $\begin{array}{l}0.42 \\
0.18\end{array}$ & $\begin{array}{l}0.31 \\
0.82\end{array}$ & $\begin{array}{l}4.72 \\
5.25\end{array}$ & $\begin{array}{l}1.05 \\
0.91\end{array}$ \\
\hline Italy & $\begin{array}{l}M \\
W\end{array}$ & $\begin{array}{r}965 \\
1016\end{array}$ & $\begin{array}{l}73.80 \\
73.87\end{array}$ & $\begin{array}{l}85.49 \\
63.68\end{array}$ & $\begin{array}{l}1.04 \\
0.20\end{array}$ & $\begin{array}{l}0.62 \\
0.10\end{array}$ & $\begin{array}{l}3.42 \\
4.82\end{array}$ & $\begin{array}{l}1.35 \\
1.18\end{array}$ \\
\hline France & $\begin{array}{l}M \\
W\end{array}$ & $\begin{array}{l}1195 \\
1626\end{array}$ & $\begin{array}{l}74.67 \\
76.03\end{array}$ & $\begin{array}{l}73.05 \\
45.26\end{array}$ & $\begin{array}{l}0.17 \\
0.12\end{array}$ & $\begin{array}{l}1.59 \\
0.43\end{array}$ & $\begin{array}{l}6.03 \\
4.86\end{array}$ & $\begin{array}{l}5.52 \\
8.36\end{array}$ \\
\hline Denmark & $\begin{array}{l}M \\
W\end{array}$ & $\begin{array}{l}487 \\
558\end{array}$ & $\begin{array}{l}73.68 \\
75.27\end{array}$ & $\begin{array}{l}71.87 \\
53.05\end{array}$ & $\begin{array}{l}0.62 \\
0.00\end{array}$ & $\begin{array}{l}0.41 \\
0.36\end{array}$ & $\begin{array}{r}5.54 \\
4.12\end{array}$ & $\begin{array}{r}9.03 \\
10.75\end{array}$ \\
\hline Switzer-land & $\begin{array}{l}\text { M } \\
\text { W }\end{array}$ & $\begin{array}{l}858 \\
958\end{array}$ & $\begin{array}{l}73.35 \\
74.34\end{array}$ & $\begin{array}{l}77.74 \\
57.93\end{array}$ & $\begin{array}{l}0.58 \\
0.00\end{array}$ & $\begin{array}{l}1.86 \\
1.46\end{array}$ & $\begin{array}{l}4.31 \\
5.85\end{array}$ & $\begin{array}{l}5.48 \\
8.87\end{array}$ \\
\hline Belgium & $\begin{array}{l}M \\
W\end{array}$ & $\begin{array}{l}1052 \\
1321\end{array}$ & $\begin{array}{l}74.60 \\
75.58\end{array}$ & $\begin{array}{l}71.10 \\
47.62\end{array}$ & $\begin{array}{l}2.76 \\
1.74\end{array}$ & $\begin{array}{l}1.52 \\
1.36\end{array}$ & $\begin{array}{l}4.18 \\
4.09\end{array}$ & $\begin{array}{r}6.56 \\
7.12\end{array}$ \\
\hline Czech Republic & $\begin{array}{l}M \\
W\end{array}$ & $\begin{array}{l}1281 \\
1726\end{array}$ & $\begin{array}{l}72.94 \\
73.48\end{array}$ & $\begin{array}{l}77.83 \\
45.42\end{array}$ & $\begin{array}{l}0.16 \\
0.06\end{array}$ & $\begin{array}{l}1.17 \\
0.64\end{array}$ & $\begin{array}{l}2.58 \\
1.68\end{array}$ & $\begin{array}{r}5.62 \\
11.70\end{array}$ \\
\hline Poland & $\begin{array}{l}M \\
W\end{array}$ & $\begin{array}{l}398 \\
459\end{array}$ & $\begin{array}{l}73.61 \\
74.31\end{array}$ & $\begin{array}{l}73.87 \\
52.72\end{array}$ & $\begin{array}{l}0.00 \\
0.00\end{array}$ & $\begin{array}{l}2.51 \\
1.53\end{array}$ & $\begin{array}{l}1.26 \\
2.40\end{array}$ & $\begin{array}{l}0.50 \\
1.74\end{array}$ \\
\hline Hungary & $\begin{array}{l}M \\
W\end{array}$ & $\begin{array}{l}591 \\
789\end{array}$ & $\begin{array}{l}73.05 \\
73.93\end{array}$ & $\begin{array}{l}78.34 \\
45.88\end{array}$ & $\begin{array}{l}1.86 \\
0.51\end{array}$ & $\begin{array}{l}0.17 \\
0.25\end{array}$ & $\begin{array}{l}3.72 \\
1.52\end{array}$ & $\begin{array}{r}4.40 \\
7.22\end{array}$ \\
\hline Portugal & $\begin{array}{l}\text { M } \\
\text { W }\end{array}$ & $\begin{array}{l}453 \\
540\end{array}$ & $\begin{array}{l}72.60 \\
73.69\end{array}$ & $\begin{array}{l}84.11 \\
59.07\end{array}$ & $\begin{array}{l}0.66 \\
0.74\end{array}$ & $\begin{array}{l}1.32 \\
1.48\end{array}$ & $\begin{array}{l}1.32 \\
4.26\end{array}$ & $\begin{array}{l}4.19 \\
3.52\end{array}$ \\
\hline Slovenia & $\begin{array}{l}\text { M } \\
\text { W }\end{array}$ & $\begin{array}{l}565 \\
741\end{array}$ & $\begin{array}{l}73.83 \\
74.79\end{array}$ & $\begin{array}{l}83.54 \\
50.20\end{array}$ & $\begin{array}{l}1.95 \\
1.35\end{array}$ & $\begin{array}{l}0.53 \\
0.40\end{array}$ & $\begin{array}{l}2.48 \\
3.78\end{array}$ & $\begin{array}{l}1.59 \\
2.97\end{array}$ \\
\hline Estonia & $\begin{array}{l}\mathrm{M} \\
\mathrm{W}\end{array}$ & $\begin{array}{l}1433 \\
2291\end{array}$ & $\begin{array}{l}73.90 \\
74.87\end{array}$ & $\begin{array}{l}80.25 \\
43.21\end{array}$ & $\begin{array}{l}0.35 \\
0.26\end{array}$ & $\begin{array}{l}1.67 \\
1.27\end{array}$ & $\begin{array}{l}4.19 \\
6.72\end{array}$ & $\begin{array}{l}5.23 \\
9.78\end{array}$ \\
\hline
\end{tabular}

Note: M - Men, W - Women, M-LT - Married living together, RP - Registered partnership, M-LS - Married, living separated, S - Single, D - Divorced, W - Widowed, ND - Not available, P - Primary or first stage of basic education, LS - Lower secondary, or second stage of basic education, US - Upper secondary, PS-NT - Post-secondary non-tertiary, 1S-T - First stage of tertiary, 2S-T - Second stage of tertiary, O+ND - Other, pre-primary, no degree and not available.

Source: based on Share 4, Release 1.1.1. 


\begin{tabular}{|c|c|c|c|c|c|c|c|c|}
\hline \multicolumn{2}{|c|}{ Marital status (in \%) } & \multicolumn{7}{|c|}{ Education - ISCED (in \%) } \\
\hline W & ND & $P$ & LS & UP & PS-NT & $1 S-T$ & $2 S-T$ & $\mathrm{O}+\mathrm{ND}$ \\
\hline 9.28 & 1.80 & 12.07 & 5.41 & 44.95 & 4.68 & 26.49 & 4.32 & 2.07 \\
\hline 37.86 & 0.99 & 23.02 & 16.62 & 37.01 & 3.30 & 16.69 & 1.78 & 1.58 \\
\hline 8.48 & 2.83 & 0.00 & 4.65 & 52.73 & 3.43 & 35.15 & 0.00 & 4.04 \\
\hline 23.55 & 1.93 & 0.00 & 25.48 & 52.46 & 1.28 & 17.77 & 0.00 & 3.00 \\
\hline 8.71 & 2.64 & 31.42 & 11.20 & 10.89 & 5.75 & 17.57 & 0.00 & 23.17 \\
\hline 19.89 & 1.86 & 28.33 & 13.88 & 11.73 & 7.73 & 17.60 & 0.00 & 20.74 \\
\hline 9.56 & 3.64 & 12.44 & 29.29 & 25.64 & 0.00 & 27.01 & 0.00 & 5.61 \\
\hline 25.21 & 2.05 & 16.99 & 48.08 & 18.22 & 0.00 & 12.74 & 0.00 & 3.97 \\
\hline 7.14 & 4.20 & 47.22 & 15.01 & 6.09 & 0.00 & 5.98 & 0.00 & 25.71 \\
\hline 28.08 & 3.26 & 52.08 & 11.50 & 2.90 & 0.00 & 3.80 & 0.00 & 29.71 \\
\hline 6.94 & 1.14 & 52.95 & 21.76 & 14.40 & 1.55 & 4.66 & 1.14 & 3.52 \\
\hline 29.23 & 0.79 & 62.99 & 13.78 & 10.63 & 2.17 & 3.25 & 0.10 & 7.09 \\
\hline 11.97 & 1.67 & 32.89 & 5.19 & 28.87 & 0.00 & 13.72 & 3.68 & 15.65 \\
\hline 39.24 & 1.72 & 40.47 & 9.16 & 18.94 & 0.00 & 10.46 & 1.78 & 19.19 \\
\hline 10.88 & 1.64 & 13.55 & 1.64 & 47.43 & 0.00 & 35.73 & 0.00 & 1.64 \\
\hline 31.00 & 0.72 & 25.81 & 8.96 & 35.66 & 0.00 & 27.96 & 0.00 & 1.61 \\
\hline 7.81 & 2.21 & 16.78 & 27.74 & 36.60 & 2.21 & 14.34 & 0.00 & 2.33 \\
\hline 25.05 & 0.84 & 13.88 & 27.45 & 41.65 & 3.13 & 12.94 & 0.00 & 0.94 \\
\hline 13.31 & 0.57 & 17.21 & 23.10 & 26.62 & 0.38 & 28.99 & 0.10 & 3.61 \\
\hline 37.09 & 0.98 & 18.55 & 22.86 & 26.87 & 0.15 & 28.31 & 0.08 & 3.18 \\
\hline 11.32 & 1.33 & 13.43 & 17.41 & 41.45 & 11.94 & 13.19 & 0.00 & 2.58 \\
\hline 39.51 & 0.98 & 11.30 & 16.34 & 45.65 & 10.43 & 14.08 & 0.00 & 2.20 \\
\hline 12.06 & 9.80 & 41.46 & 0.00 & 30.90 & 5.03 & 9.05 & 0.00 & 13.57 \\
\hline 36.38 & 5.23 & 60.35 & 0.44 & 22.88 & 2.40 & 3.05 & 0.00 & 10.89 \\
\hline 11.17 & 0.34 & 1.18 & 26.90 & 40.61 & 9.14 & 21.32 & 0.00 & 0.85 \\
\hline 44.61 & 0.00 & 3.80 & 50.32 & 29.91 & 3.68 & 12.04 & 0.00 & 0.25 \\
\hline 8.17 & 0.22 & 50.33 & 8.39 & 5.52 & 1.10 & 24.28 & 1.77 & 8.61 \\
\hline 30.93 & 0.00 & 49.07 & 7.78 & 2.96 & 0.00 & 21.48 & 0.19 & 18.52 \\
\hline 9.20 & 0.71 & 6.90 & 15.93 & 50.44 & 0.00 & 19.47 & 0.53 & 6.73 \\
\hline 41.16 & 0.13 & 9.45 & 36.84 & 33.06 & 0.00 & 11.07 & 0.13 & 9.45 \\
\hline 8.30 & 0.00 & 10.05 & 32.24 & 25.54 & 10.68 & 19.40 & 1.95 & 0.14 \\
\hline 38.67 & 0.09 & 10.00 & 30.21 & 26.36 & 15.15 & 17.20 & 0.83 & 0.26 \\
\hline
\end{tabular}


a)
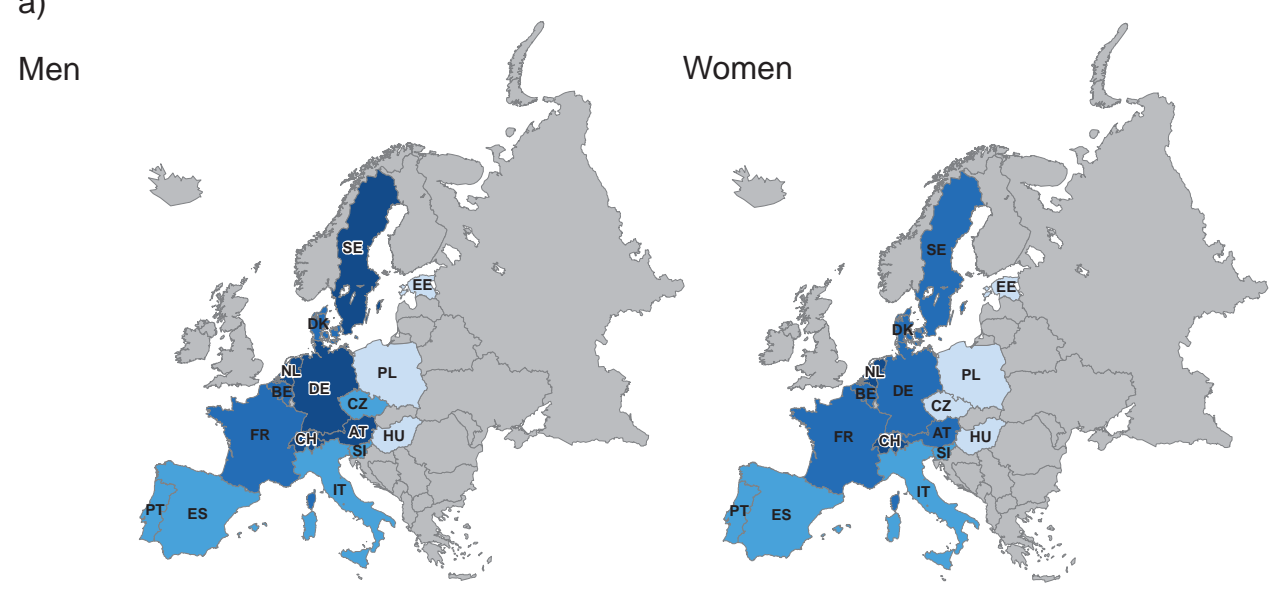

$\therefore$

Material dimension

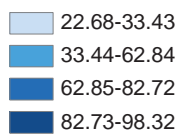

b)
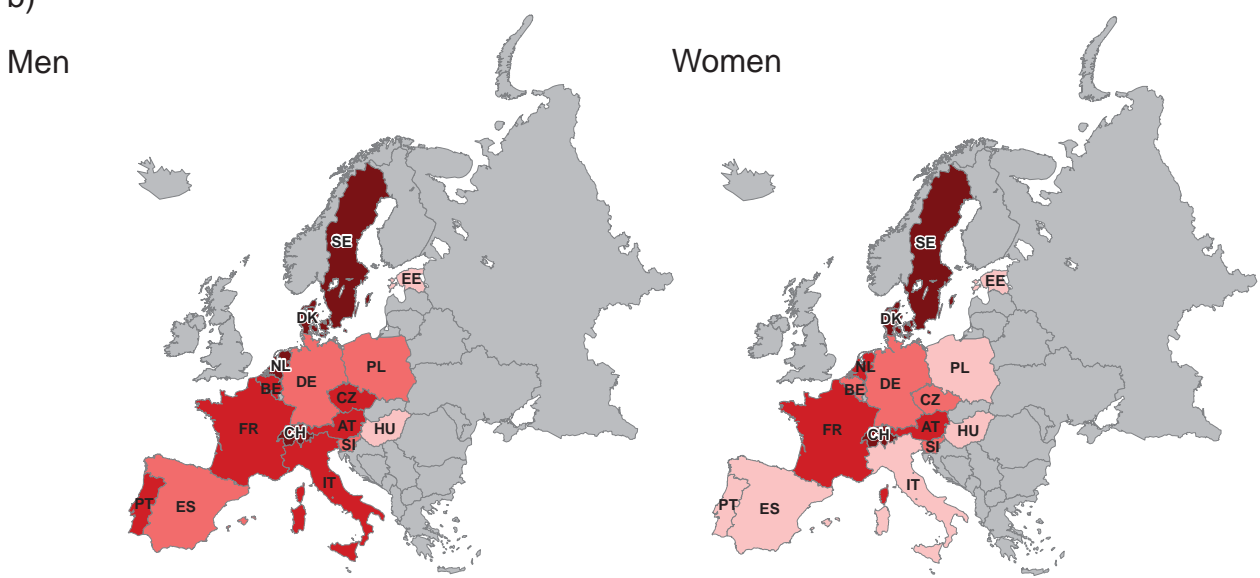

$\therefore$

Health dimension

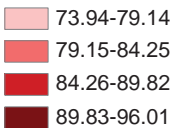

country boundary

data not available

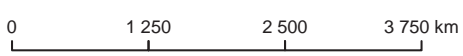

Source: own calculation based on SHARE 4 Release 1.1.1, Eurostat 2014, WHO 2014.

Figure 1a-b. Material, Health, Social and Emotional dimension of seniors Europe 
c)
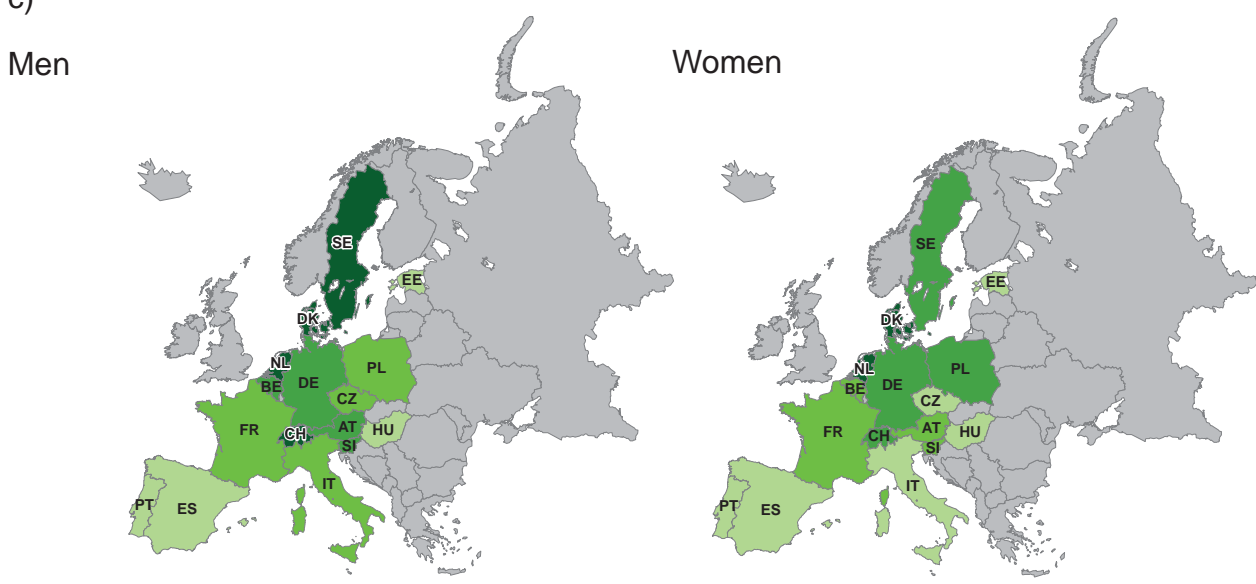

$\therefore \infty$

\section{Social dimension}

60.14-68.88

$68.89-79.09$

$79.10-87.29$

87.30-97.05

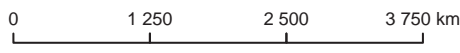

Source: own calculation based on SHARE 4 Release 1.1.1, Eurostat 2014, WHO 2014.

d)
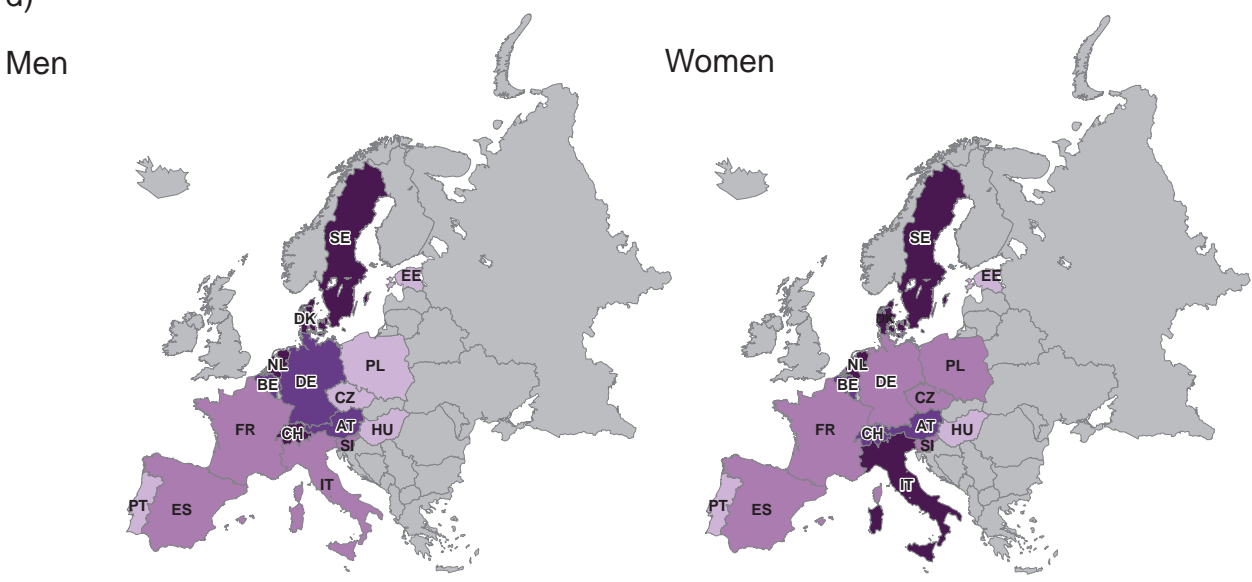

$\therefore \rho$

r.w

\section{Emotional dimension}
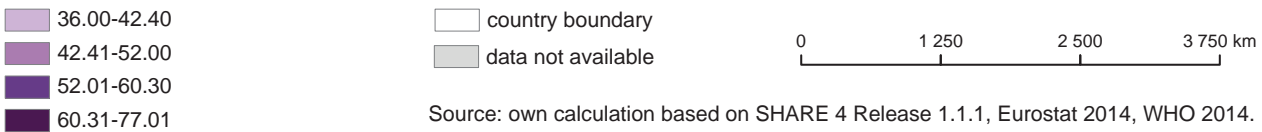

Source: own calculation based on SHARE 4 Release 1.1.1, Eurostat 2014, WHO 2014.

Figure 1c-d. Material, Health, Social and Emotional dimension of seniors Europe 
(1999) who highlighted the fact that the vulnerable or marginal position of (single) female seniors in the Netherlands was such that most women over the age of 50 did not have their own job-related income, and many were largely or totally dependent on national pensions arrangements, and lived on average in lower quality housing than senior men. However, under the name "Grey your own Way", the management board of the Dutch Policy on the Elderly subsidised a large national project from 1995 to 1997 with the aim of increasing the well-being and capacities of older women for and by women. In response to this, the Netherlands eliminated this disparity and Dutch women now receive an amount that is comparable with that received by men.

\section{Health dimension}

Health was the most frequently characterised indicator when assessing quality of life. It plays an important role in the continuing ageing process. "At the age of 60-74 up to $80 \%$ of individuals have a chronic disease, above 70 it is up to 87\%" (Dvořáčková, 2012). Similar values were also stated by Marengoni et al. (2008), in which they pointed out that $30.5 \%$ have one chronic disease and up to $54.8 \%$ have more than one chronic disease (so-called multi-morbidity). Thus, this is a factor that clearly affects the quality of life of seniors.

Figure $1 \mathrm{~b}$ presents this dimension, in which men above 65 living in European countries generally gave their health condition a better rating than women of the same age. This may seem paradoxical, as women on average live longer than men, and it might be expected that they would rate their health condition more positively. However, despite the fact that women live longer, they have chronic conditions that contribute to their more negative evaluation of their health (Kirchengast \& Haslinger, 2008). This could result from different biological predispositions or different social and gender roles in society (Orfila et al., 2006). A higher incidence of arthritis and depression among senior women has been proven by research (Alonso et al., 2004; Haro et al., 2006) and these conditions, as well as osteo-muscular conditions most negatively affect their quality of life. Additionally, men have a more superficial attitude toward their health; therefore their health evaluation may not be as thorough as that of women. Moreover, if first symptoms are ignored, health problems are identified at a later stage and cause higher mortality in the male population.

A comparison of individual countries showed very small differences, because with age, bodies become more fragile and prone to disease, irrespective of the geographical dimension. Nevertheless, clusters of more favourable regions could be identified. The best rating of this dimension came from seniors, both men and women, living in Sweden, Switzerland and Denmark. On the other hand, the lowest scores came from seniors above 65 from Hungary, Estonia and Slovenia, and the trio of the lowest values came from women seniors in Portugal, Hungary and Estonia. It is extremely challenging to identify the impacts affecting seniors' evaluation of their health. Of all indicators, healthy years of life among the seniors had the biggest variation spread, followed by functional limitations. By analysing the different levels of satisfaction or health of seniors above 65 , we considered the impacts of the level of "development" and availability of health care, purchasing power (GDP) of the population, as well as the social awareness about health (prevention) in the relevant country. We identified a strong linear relationship between health-care expenditure and the relevant dimension $(r=0.887$ in men and $r=0.844$ in women), which is statistically significant at the level of $\alpha=5 \%$. GDP effects have been identified by Beckfield (2004) and Castilla (2004) in their work, in which people living in wealthier countries are healthier but the line is curvilinear. Seniors, both men and women, from countries in Northern and Western Europe evaluated their health as much better. This, to a significant degree, relates to the highest education achieved. As shown in Tab. 4, Southern and Eastern Europe 
countries had a significantly higher number of seniors with a lower level of education. Education is one of the most important lifestyle factors and its impact is more significant, particularly in Eastern European countries (Von Dem Knesebeck, Verde, \& Dragano, 2006). Actually, Olsen and Dahl (2007) stated that lifestyle is the most important health factor in Eastern Europe. Thus, an individual approach (e.g. consumption of red meat, alcohol versus fruits and vegetables, physical activity, interest in preventive check-ups) can be considered as an equally crucial factor. From the institutional aspect, we also considered the influence of national healthcare systems. European countries can be divided into two groups when it comes to the basic perception. The 'Beveridge Model' is characterised by healthcare covered from the state budget (collection through taxes). Co-pay of patients exists, but it is low and often accompanied by deductions for certain groups (apart from others for seniors). From among the examined countries, this is applied in Sweden, Denmark, Italy, Greece, Spain and Portugal. The other system is the 'Bismarck Model' based on the system of health insurance (paid by the employer and the employee). The ageing of the population increases the pressure on expenditures and thus on the co-pay of the patient. It is applied in France, Belgium, the Netherlands, Switzerland, Germany, Austria, the Czech Republic, Poland and Hungary. Both systems however guarantee minimum healthcare, but with problematic long waiting periods. Therefore, the division of countries based on the healthcare system does not seem to be significant. The equipment, innovation in terms of medicaments, the number of healthcare facilities and staff, which is very closely related to the economic situation, are more important issues. The significance of the economic situation and the availability of healthcare were also considered to be important factors in the studies by Dragomirecká et al. (2008) and von dem Knesebeck et al. (2006). Proof of this is the position of Switzerland, where the above dimension clearly had the highest score. Switzerland's health-care expenditures are among the highest in the world (only the United States, the Netherlands, France, Germany and Canada spent a larger share of GDP on healthcare in 2011) (Thomson, Osborn, \& Squires, 2013).

\section{Social dimension}

"Social networks are defined as stable but evolving relational fabrics constituted by family members, friends and acquaintances, work and study connections, and relations that evolve out of each individual participation in formal and informal organizations. Inside any social network, nodes are linked by relationships of reciprocity. People embedded in social networks are strengthened in the social role and are likely to have lower mortality rates from cardiovascular diseases, accidents, suicides and all causes" (de Belvis et al., 2008). The significance of this dimension not only results in finding substitutes for former work roles and from the feeling of being useful; it also has a favourable impact on other dimensions. In terms of the internal structure by gender, we saw a slightly higher participation of men in economic activities, which related to their higher retirement age. On the other hand, women usually "compensate" for such loss by volunteer activities (particularly in religious organisations). Nevertheless, partial indicators of this dimension in general did not show significant gender-related differences. As already shown by past research (Olsen \& Dahl, 2007; Haski-Levetnhal, 2009) the involvement in individual countries was significantly different. In Western and Northern Europe $50-76 \%$ of seniors participated in various economic and social activities while in Southern and Eastern Europe this was only one-fourth to one-third of the senior population. The factors influencing economic activities mainly included better opportunities formed by the fight against age discrimination, the creation of part-time job opportunities, as well as higher education (Tab. 4). In this context we need to point out that this dimension is also indirectly affected by overall unemployment. The indirect 
linear correlation was $r=-0.657$ for men and $r=-0.518$ for women, and in both cases it is a statistically-significant factor. In many countries with increased economic problems and rising unemployment, firms dismiss older people first. In men, marital status was also of statistical importance (measured by establishing the percent of married); for women the same only applied in Northern and Western European countries. However, for men, the relation is indirect and for women direct. "Volunteering is a cultural and economic phenomenon, and it is part of the way societies are organized and allocate social responsibilities, and how much participation they expect from citizens. Local cultures, political climate, governmental policy, history, and norms can all impact the trends of volunteering in a country" (Haski-Leventhal, 2009). Molzahn et al. (2011) in this context, spoke about a so-called post-communist syndrome where people living in Eastern European countries insufficiently identified with their citizenship and community, were inured to their families and thus did not participate in such activities. Traditionally, people living in these countries relied more on help from the family that resulted, in many cases, from a higher level of religiosity. These findings correspond to the theory of welfare regimes, based on which, Northern European countries are classified as social-democratic with a marginal function for the institute of the family. Conversely, Eastern European countries are classified as conservative and Southern European countries, specifically Latin or South-European countries are classified as relying on the central function of the family and church (Fenger, 2007). Nevertheless, in all of the populations studied there was a high level of contact with children (80-96\%). Thus, the overall picture of the social commitment dimension corresponded more with social involvement (Fig. 1c). The highest values, for both genders, were in Sweden, Denmark and Netherlands, and, among men, also in Switzerland. On the other hand, seniors, both male and female, from Portugal, Spain, Italy, Hungary, and Estonia achieved only 52-68\% of the maximum score for this dimension.
We also see a direct lineal correlation for both genders between the health and social dimension ( $r=0.8$ for both genders). We can understand that seniors without health limitations can participate more extensively in economic and social activities (including independent transport, organisation, studies at universities of the third age), and help their children bring up their grandchildren. Good health can be considered one of the prerequisites of active ageing. On the other hand, bad health can lead to visits of family members (household care etc.), namely in the family-oriented societies of Southern and Eastern Europe.

\section{Emotional dimension}

The traditional stereotype of old age depicts a period of inevitable and continuous loss, with decreased subjective well-being. However, although negative life events tend to become more frequent and cognitive functions and health tend to decline as people get older, emotional well-being does not appear to be compromised by the ageing process (Bengtson, Gans, Putney, \& Silverstein, 2008). The dimensions mentioned above achieved a better rating in Western Europe than in Eastern Europe but the dimension of emotional well-being did not follow this trend. This might be due to the fact that this dimension is most affected by the actual emotional condition of the senior respondent. In this age group of the population, social networks, marital status, and/or other family bonds are deemed a significant factor of satisfaction within the emotional dimension of life (Haski-Leventhal, 2009). This is because people with good social relations feel safe if care or other help or assistance becomes necessary. Moreover, they feel useful, a feeling that is usually lost when retiring from a job. As mentioned above, the seniors of Northern and Western Europe were much better in this respect. However, there are other factors, such as health, education, and economic income that are reflected in one's mental condition. In particular, financial circumstances could be deemed the decisive factor 
in the allocation of the values of the prosperity indicator, where seniors from Eastern and Southern Europe, due to a fear for the future, showed much lower values than the population of Northern and Western Europe. This was also confirmed by the positive and statistically-significant correlation with GDP at values of 0.855 in men and 0.647 in women. For women, a correlation with the age of the respondent $(r=0.743)$ and her marital status ( $r=0.446)$ was observed, particularly for women of Southern and Eastern Europe $(r=0.538)$. It became apparent that this dimension was also closely linked to the respondent's health. The relationship to the health dimension was statistically significant $(\alpha=5 \%)$ and strongly linear for both genders ( $r=0.821$ men, $r=0.763$ women).

Moreover, contrary to previous evaluations, there was no correlation with the geographical picture by gender. Aurelia and Baldazzi (2002) used the Italian population as an example to show that men's dissatisfaction grew with their health problems and women were at a significant poverty risk. Gender is a specific factor in terms of predisposition for certain diseases. Depression is more typical in women, which was also apparent in our survey. This was significantly affected by their marital status; the incidence of widows was much higher than the incidence of widowers of the same age (Aurelia \& Baldazzi, 2002; Kim \& Kim, 2003). A significant change in the spatial formula of satisfied and less satisfied seniors was also noted in another partial indicator - the suicide rate. This indicator showed that Southern European countries (Spain, Italy) and the Netherlands had the lowest suicide rate among seniors, both men and women. On the other hand, the situation was the worst for senior women in Switzerland and senior men in Hungary, who had the highest suicide rate. The reason for the worst score in Switzerland is perhaps caused by liberal legislation allowing voluntary euthanasia, which is documented also by an unreasonably high value for Swiss men. The same option also exists in the Netherlands and Belgium, but only Swiss law allows foreigners to take advantage of assisted suicide, perhaps resulting in "suicide tourism".

The most satisfied seniors in terms of the emotional dimension lived in the Netherlands, Denmark and Switzerland, and senior women in Sweden and Denmark, and in the Netherlands, particularly with respect to their high score in indicators of the percentage of the population not in depression and the percentage of those who are thriving (Fig. $1 \mathrm{~d}$ ).

\section{Aggregate quality of life index}

The evaluation of individual dimensions provides detailed information on various aspects and their regional specifics. Sometimes, the spatial picture of the dimensions differed. The aggregate quality of life index allowed us to make synthesized conclusions concerning the living standards of seniors in Europe. The values of the quality of life index varied from 39 points for men and 38 points for women. Men had slightly higher scores, except in Poland and Sweden where no gender differences were identified. In these two countries, men reported a better score in the economic dimension while the health dimension did not show significant differences, while women dominated in the emotional domain and Polish women dominated in social engagement. However, the largest difference in favour of men (4-5 percentage points) was recorded mainly in German-speaking countries such as Germany, Austria and Switzerland as well as in Portugal. In these countries, women were surpassed by men in all the monitored dimensions.

In terms of region, the aggregate quality of life index defined the central part of Western and Northern Europe (Fig. 2, Tab. 2) as the focal area with the highest satisfaction of seniors. The best rating in the aggregate quality of life index, for both men and women, was achieved by three countries, the Netherlands, Denmark and Switzerland, followed by Sweden, and all four countries had scores exceeding 80 for both men and women. These were the countries which, in terms of partial dimensions, had achieved the maximum 

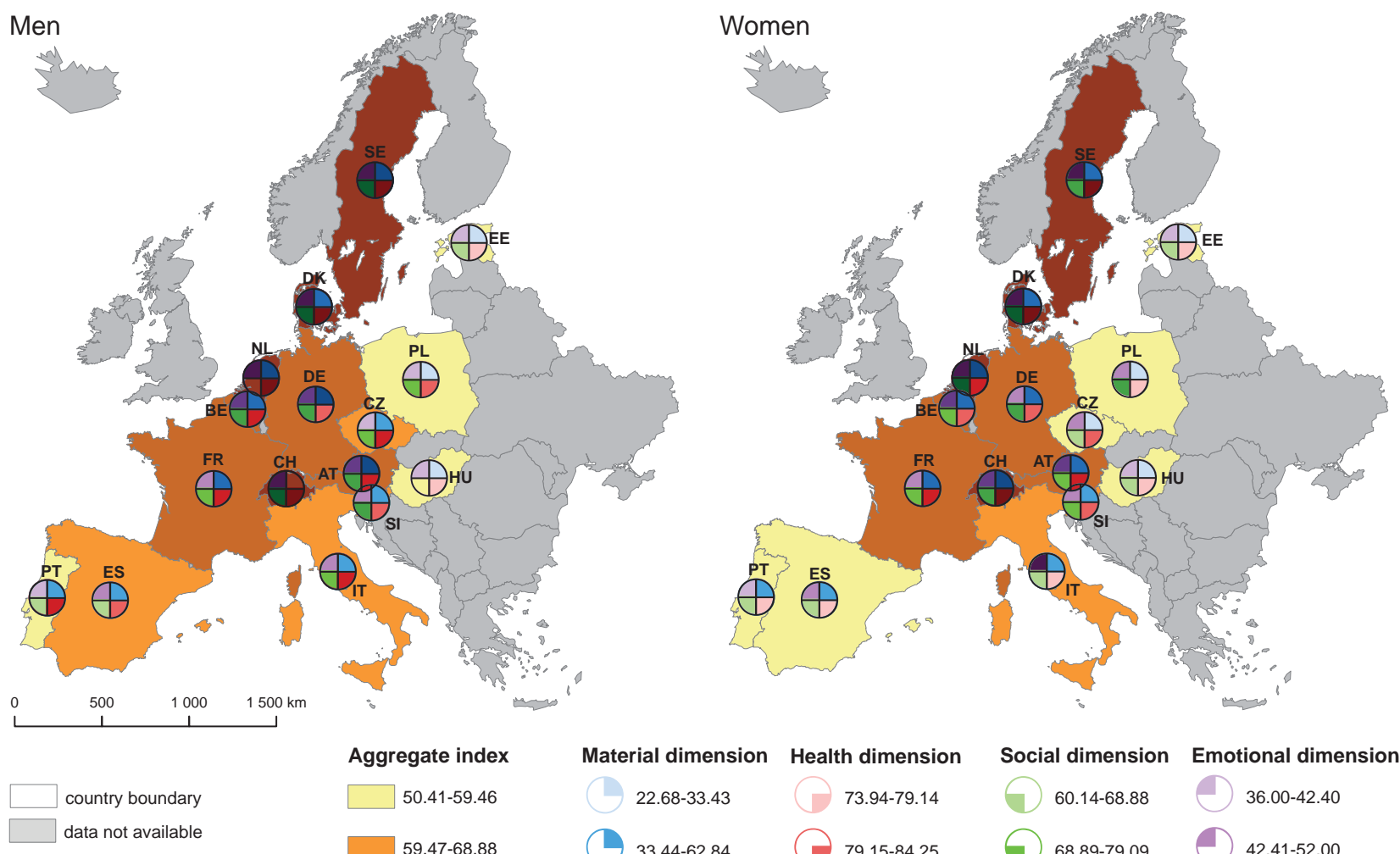

Dimension of well-being

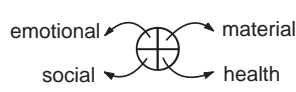

Aggregate index

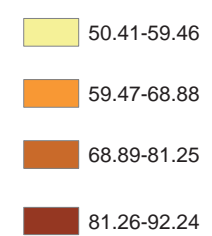

Material dimension Health dimension

22.68-33.43 73.94-79.14

$33.44-62.84$

$\bigcirc 62.85-82.72$

82.73-98.32

(79.15-84.25

(1) $84.26-89.82$

(P).83-96.01
Social dimension Emotional dimension

(1) $60.14-68.88 \bigcirc 36.00-42.40$

68.89-79.09

79.10-87.29

( $87.30-97.05$

$42.41-52.00$

(52.01-60.30

C6.31-77.01

Source: own calculation based on SHARE 4 Release 1.1.1, Eurostat 2014, WHO 2014

Figure 2. Aggregate quality-of-life index of seniors Europe 
values or ranked in leading positions. The high level of satisfaction of (not only) seniors in these countries within the surveyed areas of their lives resulted from the overall standard in the economic, social, and health areas. The second side of the spectrum defines the countries with the lowest total score on the aggregate index. These ranks were taken for both senior women and men by the countries of Estonia, Hungary and Portugal - particularly because of significantly undersized values in the material and emotional fields. The values of the synthesized index of these countries ranged from 50 to 59 , thus only reaching fifty percent of the highest possible quality of life currently in the European area.

A look at Tab. 2 reveals that in terms of diversification of the quality of life index, the material dimension was the most significant. The values indicated the biggest differences between the evaluations of seniors from individual countries. On the other hand, the ranking of the emotional dimension indicated the highest internal heterogeneity of individual indicators. Also, the countries that ranked best had relatively high reserves in certain indicators of this dimension.

\section{Conclusion}

Quality of life is a complex phenomenon comprising several aspects involving biological, psychological, economic, aesthetic, social, ethical, philosophical, and other dimensions (Uher, 2014). For a specific population group, and seniors are definitely such a group, the evaluation of quality of life is a much more sensitive issue.

Previous scientific studies and international reports offer a wide range of research in terms of diversity in the field of quality of the life of seniors; this is related to its extraordinarily multi-dimensional nature. Based on their results we cannot identify the single most significant dimension determining the quality of life of seniors. The core of the quality of life of seniors was determined by the aim of the given research. Therefore, we chose a complex approach in our study. Our research points out the fact that some dimensions are closely related (the health and social dimensions). However, the economic dimension can be considered as decisive when it comes to the overall quality of life. Both partial indicators reach high values in the correlation matrix. Seniors with a higher income can live emotionally more relaxed lives, their nutrition is better and consequently thanks to good health they can lead socially active lives.

According to the analysed European survey of seniors' evaluation of the quality of their lives, seniors living in Western and Northern European countries are more satisfied with their quality of life. These countries provide institutional support, be it in the field of health care or social infrastructure. Also significant were the effects of a positive correlation with the country's advanced economy. The quality of life of these citizens resulted as well from long-term (lifelong) individual preparation for the time (age) of becoming a senior. People in Northern and Western European countries traditionally (historically) have relied to a larger extent on institutional care. In the social-democratic welfare regime, the provision of social welfare was delegated to the state. Citizens lived together within their community and participated in voluntary activities, which resulted in a higher quality of social engagement. They were also more responsible regarding finances and living healthier lifestyles.

Countries in Southern and Eastern Europe had lower values in individual dimensions as well as in the aggregate quality of life index. The most significant differences were documented in the material dimension. Individual factors might also be considered with the total socioeconomic standing of the countries (particularly a high unemployment rate). Seniors in Southern and Eastern Europe have traditionally have relied on help from family, which has allowed them to adopt a passive approach to growing older. Problems in these countries may even grow due to the falling birth rate and the fact that families are becoming smaller. 
In terms of gender structure, men more frequently declared being satisfied with their quality of life. This fact was closely related to objective factors - higher income throughout the life and marital status (a lower number of widowed individuals), particularly in Southern and Eastern Europe. Moreover, certain gender roles and stereotypes have been adopted requiring that men should act as the strong ones providing income without complaining about their health. It can be assumed that there is probably a subjective aspect.

Globally, quality of life appears to be interlinked with the institutional framework of the country, the support received from the family and individual approaches. Longterm preparation for older age at all levels seems to be a necessary aspect of quality of life in the future. Income is one of the most important factors of quality of life. It is significantly influenced by the country's GDP, but individual factors should not be omitted. Responsible preparation for this stage of life should include savings and if allowed under the legislative framework, diversifying one's income sources. In this context, Šlapák and Soukup (2008) reported that only 53\% of the Czech population actively spend time to provide for their finances in the last two years before retiring. This was also confirmed by the fact that only 83,000 individuals had opted to participate in the second pension pillar in the Czech Republic. Such undersized preparation for growing old cannot be reversed at a later point in time.

The quality of life also significantly improves if seniors have sufficient social networks. Thus, creating conditions for part-time jobs, accessible public spaces and transportation, supporting active ageing through education, cultural and sport events should be a goal of each country, city and community. Such conditions will not only improve the social dimension but also the emotional dimension and even the health dimension.

\section{Acknowledgements}

The paper was written within the project APVV-15-0184 entitled "Intergenerational social networks in an aging city - continuity and innovation 2016-2020".

This paper uses data from SHARE wave 4 release 1.1.1, as of March 28th 2013 or SHARE wave 1 and 2 release 2.5.0, as of May 24th 2011 or SHARELIFE release 1, as of November 24th 2010. The SHARE data collection has been primarily funded by the European Commission through the 5th Framework Programme (project QLK6-CT-2001-00360 in the thematic programme Quality of Life), through the 6th Framework Programme (projects SHARE-I3, RII-CT-2006-062193, COMPARE, CIT5- CT-2005-028857, and SHARELIFE, CIT4-CT-2006-028812) and through the 7th Framework Programme (SHARE-PREP, $N^{\circ}$ 211909, SHARE-LEAP, No 227822 and SHARE M4, N²61982). Additional funding from the U.S. National Institute on Aging (U01 AG09740-13S2, P01 AG005842, P01 AG08291, P30 AG12815, R21 AG025169, Y1-AG-4553-01, IAG BSR06-11 and OGHA 04-064) and the German Ministry of Education and Research as well as from various national sources is gratefully acknowledged (see www.share-project.org for a full list of funding institutions).

\section{Editors' note:}

Unless otherwise stated, the sources of tables and figures are the authors', on the basis of their own research. 


\section{References}

Alonso, J., Angermeyer, MC., Bernert, S., Bruffaerts, R., Brugha, T. S., Bryson, H., de Girolamo, G., ... Vollebergh, W.A.M. (2004). Prevalence of mental disorders in Europe: results from the European Study of the Epidemiology of Mental Disorders (ESEMeD) project. Acta Psychiatrica Scandinavica Supplementum, 109(420), 21-27. https://doi.org/10.1111/j.1600-0047.2004.00327.x

Annoni, P., Weziak-Bialowolska, D., Dijkstra L. (2012). Quality of life at the sub-national level: An operational example for the EU. Luxembourg: Publications Office of the European Union. https://doi.org/10.2788/70967

Aurelia, E., Baldazzi, B. (2002). Unequal perceived quality of life among elderly Italians: different satisfaction levels in selected spheres life. Social Indicators Research, 60(1): 309-334. https://doi.org/10.1023/A:1021229602404

Barnes, M., Taylor, D., Ward, L. (2013). Being well enough in old age. Critical Social Policy, 33(3): 473-493. https://doi.org/10.1177/0261018312471163

Beckfield, J. (2004). Does income inequality harm health? New cross-national evidence. Journal of Health and Social Behavior, 45(3): 231-248. https://doi.org/10.1177/002214650404500301

Bengtson, V.L., Gans, D., Putney, N.M., Silverstein, M. (2008). Handbook of theories of aging (Second Edition). New York: Springer Publishing Company.

Bowling, A., Gabriel, Z. (2004). An integrational model of quality of life in older age. Results from the ESRC/MRC HSRC quality of life survey in Britain. Social Indicators Research, 69(1): 1-36. https://doi.org/10.1023/B:SOCl.0000032656.01524.07

Campbell, A., Converse, P.E.,Rodgers, W.L. (1976). The quality of American life: Perceptions, evaluations, and satisfactions. New York: Russell Sage Foundation.

Castilla, E.J. (2004). Organizing health care: A comparative analysis of national institutions and inequality over time. International Sociology, 19(4), 403-435. https://doi.org/10.1177/0268580904047365

de Belvis, A.G., Avolio, M., Spagnolo, A., Damiani, G., Sicuro, L., Cicchetti, A., Ricciardi, W., Rosano, A. (2008). Factors associated with health-related quality of life: The role of social relationships among the elderly in an Italian region. Public Health, 122(8), 784-793.

https://doi.org/10.1016/j.puhe.2007.08.018

Demura, S., Sato, S. (2003). Relationships between depression, lifestyle and quality of life in the community dwelling elderly: A comparison between gender and age groups. Journal of Physiological anthropology and Applied Human Science, 22(3): 159-166. https://doi.org/10.2114/jpa.22.159

Dhéret, C., Zuleeg, F., Chiorean-Sime, S. (2011). Well-being 2030: A new vision for 'Social Europe'. EPC Issue Paper, 65. Retrieved from

https:/www.files.ethz.ch/isn/131292/pub_1326_epc_issue_paper_65_-_a_new_vision_for_social_europe.pdf [15 February 2020].

Dragomirecká, E., Bartoňová, J., Eisemann, M., Kalfoss, M., Kilian, R., Martiny, K., .. \& Schmidt, S. (2008). Demographic and psychosocial correlates of quality of life in the elderly from a cross-cultural perspective. Clinical Psychology and Psychoterapy, 15(3): 193-204. https://doi.org/10.1002/cpp.571

Dvořáčková, D. (2012). Kvalita života senior. Praha: Grada Publishing.

European Comission (2009). Ageing Report: Economic and budgetary projections for the EU-27 Member States (2008-2060). Joint Report prepared by the European Commission (DG ECFIN) and the Economic Policy Committee (AWG), European Economy, 2.

European Comission. (2011). Well-being in 2030: Interim findings: September 2011. Eurobarometer Qualitative Studies conducted by TNS Qual+. Retrieved from

https://ec.europa.eu/commfrontoffice/publicopinion/archives/quali/wellbeing_interim_en.pdf [20 February 2020].

Eurostat. (2014). Statistics Database. Healthy Life Years (from 2004 onwards). Retrieved from https://data.europa.eu/euodp/en/data/dataset/dDWAuSZ7JV3Sy8j7OHNeGQ [20 February 2020]. 
Farquhar, M. (1995). Elderly people's definitions of quality of life. Social Science \& Medicine, 41(10), 14391446.

Fenger, M. (2007). Welfare regimes in Central and Eastern Europe: Incorporating post-communist countries in a welfare regime typology. Contemporary Issues and ldeas in Social Sciences, 3(2), 2-30.

Fernández-Ballesteros, R. (2002). Social support and quality of life among older people in Spain. Journal of Social Issues, 58(4), 645-659. https://doi.org/10.1111/1540-4560.00282

Haro, J.M., Palacin, C., Vilagut, G., Martínez, M., Bernal, M., Luque, I., ... \& El Grupo ESEMeD-España. (2006). Prevalence of mental disorders and associated factors: results from the ESEMeD-Spain study. Medicina Clinica, 126(12), 445-451. https://doi.org/10.1157/13086324

Haski-Leventhal, D. (2009). Elderly volunteering and well-being: A cross-European comparison based on SHARE Data. Voluntas: International Journal of Voluntary and Nonprofit Organizations, 20(4), 388-404. https://doi.org/10.1007/s11266-009-9096-x

Heston, A., Summers, R., Aten B. (2012). Penn World Table Version 7.1. Center for International Comparisons of Production. Philidelphia, PA: University of Pennsylvania.

Káčerová M, Ondačková, J., Mládek, J. (2014). Time-space differences of population ageing in Europe. Hungarian Geographical Bulletin, 63(2), 177-199. https://doi.org/10.15201/hungeobull.63.2.4

Kim, I., Kim, C.S. (2003). Patterns of family support and the quality of life of the elderly. Social Indicators Research, 62-63(1), 437-454. https://doi.org/10.1023/A:1022617822399

Kirchengast, S., Haslinger, B. (2008). Gender differences in health-related quality of life among healthy aged and old-aged Austrians: Cross-sectional analysis. Gender Medicine, 5(3), 270-278. https://doi.org/10.1016/j.genm.2008.07.001

Marengoni, A., Winblad, B., Karp, A., Fratiglioni, L. (2008). Prevalence of chronic diseases and multimorbidity among the elderly population in Sweden. American Journal of Public Health, 98(7), 1198-1200. https://doi.org/10.2105/ajph.2007.121137

Mastekaasa, A., Moum, T., Næss, S. (1988). Livskvalitetsforskning. Oslo: Institutt for samfunnsforskning.

Molzahn, A.E., Kalfoss, M., Schick Makaroff, K., Skevington, S.M. (2011). Comparing the importance of different aspects of quality of life to older adults across diverse cultures. Age and Ageing, 40(2), 192-199. https://doi.org/10.1093/ageing/afq156

Mühlpachr, P. (2004). Gerontopedagogika. Brno: Masarykova univerzita.

Netuveli, G., Blane, D. (2008). Quality of life in older ages. British Medical Bulletin, 85(1), 113-126. https:// doi.org/10.1093/bmb/ldn003

Olsen, K.M., Dahl, S.Å. (2007). Health differences between European countries. Social Science \& Medicine, 64(8), 1665-1678. https://doi.org/10.1016/j.socscimed.2006.11.031

Orfila, F., Ferrer, M., Lamarca, R., Tebe, C., Domingo-Salvany, A., Alonso, J. (2006). Gender differences in health-related quality of life among the elderly: the role of objective functional capacity and chronic conditions. Social Science \& Medicine, 63(9), 2367-2380.

https://doi.org/10.1016/j.socscimed.2006.06.017

Payne, J. (2005). Kvalita života a zdraví. Praha: Triton.

Poleij, J., Kraan, H. (1999). The policy with reference to elderly women in the Netherlands. Summary of an article for UN Magazine 'Instraw'.

Population Reference Bureau (PRB). (2011). SCL/PRB index of well-being in older populations. Final Report. Global Aging and Monitoring Project. Washington DC: Population Reference Bureau.

Statistics Canada. (2014). Low income cut-offs (1992 base) after tax.

Stiglitz, J.E., Sen, A., Fitoussi, J.P. (2010). Report by the commission on the measurement of economic performance and social progress. Paris: Commission on the Measurement of Economic Performance and Social Progress.

Šlapák, M., Soukup, T. (2008). Finanční gramotnost jako součást př́pravy na stář́. Praha, VŠE v Praze, Nakladatelství Oeconomica. 
Thomson, S., Osborn, R., Squires, D. (2013). International profiles of health care systems 2013. New York: The Commonwealth Fund.

Uher, I. (2014). Determinanty kvality života seniorov. Košice: Univerzita Pavla Jozefa Šafárika v Košiciach.

Von Dem Knesebeck, O., Verde, P.E., Dragano, N. (2006). Education and health in 22 European countries. Social Science \& Medicine, 63(5), 1344-1351. https://doi.org/10.1016/j.socscimed.2006.03.043

Walker, A., (2005). A European perspective on quality of life in old age. European Journal of Ageing, 2(1), 2-12.

Westerhof, G., Dittmann-Kohli, F., Thissen, T. (2001). Beyond life satisfaction: Lay conceptions of well-being among middle-aged and elderly adults. Social Indicators Research, 56(2), 179-203. https://doi.org/10.1023/A:1012455124295.

World Health Organization (WHO). (1997). WHOQOL: Measuring Quality of Life. Geneva: WHO.

World Health Organization (WHO). (2014). Suicide. Statistics. Country reports and charts. Geneva: WHO. 